\title{
Propiedades psicométricas del Cuestionario Revisado de Percepción de ENFERMEDAD (IPQ-R) EN ADULTOS MEXICANOS CON ASMA
}

\author{
Isaías Vicente Lugo González, Margarita Fernández Vega, \\ Yuma Yoaly Pérez Bautista y Cynthia Zaira Vega Valero \\ FES Iztacala. UNAM \\ México
}

\begin{abstract}
RESUMEN
El objetivo del presente trabajo fue evaluar las propiedades psicométricas de la versión en español del Cuestionario Revisado de Percepción de Enfermedad (IPQ-R) en pacientes mexicanos con asma. Se obtuvo una muestra por conveniencia de 315 adultos, quienes respondieron un cuestionario de evaluación. Los resultados muestran que el IPQ-R cuenta con un apartado de percepción de 14 síntomas (identidad), un grupo de subescalas en las que se encuentran seis factores (percepción emocional, temporalidad, consecuencias, control personal, coherencia y control del tratamiento), un indicador (curso cíclico,) y un apartado de percepción de causas con 18 opciones. El grupo de subescalas cuenta con alfas de $0.733-0.874$, un $K M O=0.809$ y una varianza total explicada de $51.611 \%$. Se concluye que el IPQ-R es un instrumento confiable y válido para evaluar la percepción de enfermedad en pacientes con asma. Se propone la inclusión de ítems y la modificación de formas de respuesta.
\end{abstract}

Palabras Clave:

creencias cerca del asma; percepción de enfermedad; asma; confiabilidad; validez

\section{PSychometric PROPERTIES Of ILlNess PeRCEPTION QUestionNaIRE- REVISED (IPQ-R) IN MEXICANS ADULTS WITH ASTHMA}

\begin{abstract}
The aim of this study was to evaluate the psychometric properties of the Spanish version of the Illness Perception Questionnaire-Revised (IPQ-R) in Mexican patients with asthma. A sample was obtained for convenience of 315 adults who answered an evaluation battery. The results found that the IPQ-R has a perception section of 14 symptoms (identity), a group of subscales in which there are six factors (emotional perception, timeline acute/chronic, consequences, personal control, coherence and treatment control) and an indicator (timeline cyclical) and a perception of causes section with 18 options. The subscale group has alpha levels of $0.733-0.874$, a $\mathrm{KMO}=0.809$ and a total explained variance of $51.611 \%$. It is concluded that the IPQ-R is a reliable and valid instrument to evaluate the illness perception in patients with asthma. The inclusion of items and the modification of response forms are proposed.
\end{abstract}

Keywords:

beliefs about asthma; illness perception; asthma; reliability; validity

BitÁCORA DEL ARTículo:

| Recibido: 19 de diciembre de 2019 | Aceptado: 8 de Junio de 2020 | Publicado en línea: Julio-Diciembre de 2020 | 
Artículo Empírico | Propiedades psicométricas...| Lugo-González, Fernández-Vega, Pérez-Bautista ẽ Vega-Valero

\title{
Autoría y Derechos de Propiedad Intelectual
}

\section{Propiedades psicométricas del CUestionario ReVISAdo dE Percepción de EnFermedad (IPQ-R) en AdULtos MEXICANOS CON ASMA}

\author{
Isaías Vicente Lugo González, Margarita Fernández Vega, \\ Yuma Yoaly Pérez Bautista y Cynthia Zaira Vega Valero \\ FES Iztacala. UNAM \\ México
}

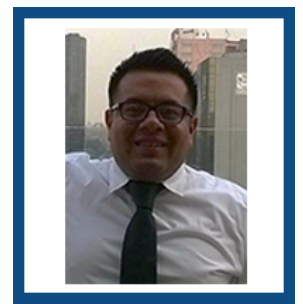

Isaías Vicente Lugo González

FES Iztacala. UNAM

Correo: isaiasvlg@comunidad.unam.mx

Estudiante del doctorado en Psicología y Salud, Facultad de Estudios Superiores Iztacala. UNAM. Docente de posgrado en Psicología. Facultad de Estudios Superiores Iztacala. UNAM.

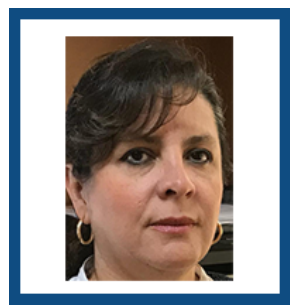

Cynthia Zaira Vega Valero FES Iztacala. UNAM Correo: vegavalero@hotmail.com

Doctora en Psicología por la UNAM, profesora titular de la carrera de Psicología y coordinadora de la residencia en Gestión Organizacional de la maestría en Psicología. de la FES Iztacala, Universidad Nacional Autónoma de México.

\section{CONTRIBUCIÓN DE LOS AUTORES}

Isaías Vicente Lugo González concibió la idea del proyecto, realizó la recolección y captura de datos, el análisis de datos y la redacción del manuscrito. I Margarita Fernández Vega supervisó la investigación, se encargo de la revisión del manuscrito y brindó asesoría especializada en asma. | Yuma Yoaly Pérez Bautista participó en la captura de datos, análisis de datos, redacción y revisión del manuscrito. I Cynthia Zaira Vega Valero concibió la idea del proyecto, participó en el análisis de datos y la revisión del manuscrito.

\section{AGRADECIMIENTOS}

Agradecimiento a Conacyt por la beca (No 303407) proporcionada al primer autor como parte de estudios doctorales.

\section{DATOS DE FiLIACIÓN DE LOS AUtORES}

FES Iztacala. UNAM | Subdirección de Enseñanza. Instituto Nacional de Enfermedades Respiratorias (INER)

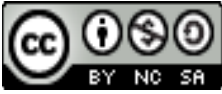

Copyright: (c) 2020 Lugo-González, I. V.; Fernández-Vega, M.; Pérez-Bautista, Y.Y. \& Vega-Valero, C.Z.

Este es un artículo de acceso abierto distribuido bajo los términos de la licencia Creative Commons Reconocimiento-NoComercial 4.0 Internacional, por lo que su contenido gráfico y escrito se puede compartir, copiar y redistribuir total o parcialmente sin necesidad de permiso expreso de sus autores con la única condición de que no se puede usar con fines directamente comerciales y los términos legales de cualquier trabajo derivado deben ser los mismos que se expresan en la presente declaración. La única condición es que se cite la fuente con referencia a la Revista Digital Internacional de Psicología y Ciencia Social y a sus autores. 


\section{TABLA DE CONTENIDO}

INTRODUCCIÓN

Objetivo, 392

MÉTODO

Participantes, 392

Diseño, 393

Instrumentos, 393

Análisis de resultados, 393

Procedimiento, 394

\section{RESULTADOS}

Análisis de confiabilidad y validez estructural, 395

Correlación entre factores, 398

Validez convergente y divergente, 398

Interpretación de las puntuaciones del IPQ-R, 399

\section{Discusión}

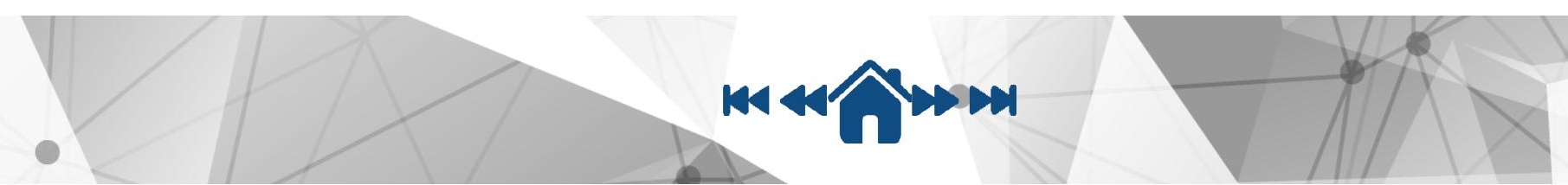




\section{INTRODUCCIÓN}

$\mathrm{E}$ I asma es una enfermedad crónica en la que se presenta inflamación de la vía aérea, obstrucción, sensibilidad e hiperreactividad a diversos elementos ambientales (Global Initiative for Asthma [GINA], 2019; Guía Mexicana de Asma [GUIMA], 2017). Se estima que en México más de nueve millones de personas padecen asma, cifra que va en aumento (Secretaría de Salud [SSA], 2016). En los últimos años el asma se ubica entre las enfermedades crónicas más atendidas en los servicios de urgencias y hospitalización (Dirección General de Epidemiología [DGE], 2015; Instituto Nacional de Enfermedades Respiratorias [INER], 2018).

Se sabe que esta continua atención de urgencia se debe, la mayoría de las veces, a problemas de adherencia y un laxo control de la enfermedad (Foster, Lavoie, y Bouler, 2011; GINA, 2016; GUIMA; 2017). Para que esto suceda están implicados diversos factores como comorbilidades (alergias, otras enfermedades respiratorias, obesidad), exposición a desencadenantes (contaminación, humo de diversas sustancias), aspectos psicosociales negativos (bajo nivel educativo, economía, depresión, ansiedad), conocimientos de la enfermedad y creencias acerca de la enfermedad, entre otras (GINA, 2019; GUIMA; 2017; Leventhal, Weinman, Leventhal, y Phillips, 2008).

En el caso del asma se ha identificado que los factores individuales como las creencias acerca de la enfermedad están vinculadas con desenlaces adversos en el manejo de la enfermedad, como los mencionados. Por ejemplo, tener una percepción de temporalidad aguda de la enfermedad, un bajo control personal, mayor percepción de síntomas, percepción negativa del tratamiento y repercusiones emocionales asociadas con la enfermedad (Achstetter, Schultz, Faller, y Schuler, 2016; Bahçecioğlu y Çevik-Akyl, 2014; Lugo-González, Reynoso-Erazo, y Fernández-Vega, 2014; Halm, Mora, y Leventhal, 2006; Sofianou et al., 2013; Unni y Shiyanbola, 2016).

Un ejemplo de lo anterior sería la indicación médica para usar el tratamiento de control diariamente, sin embargo, para algunos pacientes esta indicación carecerá de sentido usarlo así si se cree que el asma es una enfermedad cíclica o aguda y no crónica, creer que si no tienen síntomas no tienen asma (Halm, et al., 2006; Leventhal, Breland, Mora, y Leventhal, 2010; Østrem y Horne, 2015), creer que el medicamento de control sólo es necesario cuando se tiene síntomas y no lo es cuando no se presen- tan (McAndrew et al., 2008; Ponieman, Wisnivesky, Leventhal, Musumeci-Szabó, y Halm, 2009).

El ejemplo anterior se configura desde el Modelo de Sentido Común y Representación de Enfermedad (MSCRE) (Diefenbach y Leventhal, 1996), el cual se define como un modelo de autorregulación de comportamientos de salud y enfermedad. Está organizado en tres etapas, I etapa perceptual, la etapa de respuesta y la etapa de evaluación de resultados; la primera de ellas implica la evaluación de la percepción de enfermedad en dos dimensiones generales (percepción cognitiva y percepción emocional), las cuales, a su vez, se dividen en diferentes subdimensiones: identidad: experiencia perceptual de la enfermedad, tipo, lugar y cantidad de síntomas; temporalidad: percepción de duración (aguda, crónica o cíclica) de la enfermedad; causas: razones percibidas de lo que causó la enfermedad; consecuencias: repercusiones percibidas y experimentadas en diferentes áreas; control personal: capacidad percibida para controlar la enfermedad; control del tratamiento: impacto percibido que el tratamiento tendrá en el padecimiento; coherencia: claridad con que se entiende la enfermedad, y percepción emocional: percepción de repercu $\neg$ siones emocionales asociadas (Broadbent, Petrie, Main y Weinman, 2006; Cameron y Moss-Morris, 2004; Diefenbach y Leventhal, 1996; Mora y McAndrew, 2013; Moss-Morris et al., 2002).

Desde esta lectura es importante la evaluación de las subdimensiones del MSCRE para identificar cómo cada persona percibe su enfermedad y trazar la asociación con desenlaces en la enfermedad. Para hacer dicha evaluación hay diversos modos, como 1) ítems únicos, diseñados con base en el proceso del MSCRE, dirigidos a una enfermedad específica, y que implican un análisis individualizado de la percepción de la enfermedad (Mora y McAndrew, 2013; Pacheco et al., 2012); 2) dibujos que permiten identificar las percepciones y atribuciones de la enfermedad y los síntomas, además del efecto percibido de los tratamientos médicos en la condición o sintomatología de los pacientes (Cheung, Saini, y Smith, 2017; Petrie y Weinman, 2006; Petrie, y Weinman, 2012); y 3) instrumentos de evaluación, que tienen un conjunto más amplio de ítems para evaluar el nivel que hay en cada una de las subdimensiones del MSCRE (Mora y McAndrew, 2013; Moss-Morris et al., 2002; Weinman, Petrie, Moss-Morris, y Horne, 1996).

Respecto de los instrumentos de evaluación, al inicio se desarrolló el Cuestionario de Percepción de Enfermedad (IPQ) (Weinman et al., 1996), para la evaluación de la percepción cognitiva de la enfermedad, constituido por 38 ítems divididos en cinco subdimen- 
siones: identidad, causas, temporalidad, consecuencias y control-cura. En seguida se hizo una versión revisada de este instrumento (IPQ-R) (Moss-Morris et al., 2002), que ya incluyó la evaluación de la percepción emocional de la enfermedad y se conformó por 70 ítems divididos en tres partes: 1) evaluación de identidad; 2) escalas de control personal, control del tratamiento, temporalidad aguda/crónica, curso cíclico, consecuencias, percepción emocional y coherencia, y 3) escala de causas. EI IPQ-R fue validado en un grupo de 711 participantes con asma, diabetes, artritis reumatoide, dolor crónico y agudo, infarto al miocardio, esclerosis múltiple e infección por VIH. Tiene evidencias de validez estructural, divergente y predictiva, además de consistencia interna y estabilidad temporal como datos de confiabilidad.

Por último, se elaboró el Cuestionario Breve de Percepción de Enfermedad (BIPQ, Broadbent et al., 2006) para la evaluación de la percepción cognitiva y emocional de la enfermedad; está constituido por ocho ítems valorativos (0-10) divididos de la siguiente manera: 1 ) consecuencias, 2) temporalidad, 3) control personal, 4) control del tratamiento, 5) identidad, 6) preocupación, 7) coherencia, 8) representación emocional y 9) causas de la enfermedad (pregunta abierta acerca de las tres principales causas atribuidas a la enfermedad).

Actualmente el BIPQ y el IPQ-R son los instrumentos más utilizados para la evaluación de la percepción de enfermedad, con base en el MSCRE, en el contexto clínico y de investigación (Broadbent et al., 2015). Sobre todo este último ha sido adaptado a diferentes contextos y padecimientos; como ejemplo de ello se encuentran versiones para enfermedades crónicas (Aberkane, 2017), infección por VIH (Wu et al., 2018), diabetes tipo 2 (Abubakari et al., 2012; Brzoska, Yilmaz, Sultanoglu, Sultanoglu, y Razum, 2012), enfermedades cardiacas (Brink, Alsén, y Cliffordson, 2011; Brzoska et al., 2012; Chen, Tsai, y Lee, 2008), enfermedad renal crónica (Chilcot, Norton, Wellsted, y Farrington, 2012), cáncer (Dempster y McCorry, 2012; Giannousi, Manaras, Georgoulias, y Samonis, 2010; Moon, Moss-Morris, Hunter, y Hughes, 2017), dermatitis atópica (Wittkowski, Richards, Williams, y Main, 2008) y fibromialgia (Van Ittersum, Van Wilgen, Hilberdink, Groothoff, y Van der Schans, 2009).

En idioma español se identifican dos versiones para pacientes con hipertensión (Beléndez, Bermejo, y García, 2005; Pacheco et al., 2012) en las que se mantiene la estructura factorial de la versión original; sin embargo, en la de Beléndez et al. (2005) el número de ítems está modificado debido al proceso de análisis psicométrico; por lo contrario, el propósito del estudio de Pacheco et al. (2012) fue hacer la adaptación y traducción del IPQ-R en su ver- sión original, por lo que es similar en cuanto a estructura, contenido de ítems y número de éstos. En el caso de México, hay una versión modificada del IPQ-R y adaptada para personas con obesidad (Hurtado-Piña, 2017). Al ser una herramienta de amplia aplicación, y que permite el conocimiento de la percepción que los pacientes tienen de su enfermedad, es importante contar con estos instrumentos para la evaluación de este tipo de variables, así como evaluar los efectos de programas de intervención dirigidos a modificar este tipo de elementos.

\section{Objetivo}

Este trabajo tuvo como propósito evaluar las propiedades psicométricas de consistencia interna y estructura factorial de la versión en español del IPQ-R en adultos mexicanos con asma.

\section{MÉTodo}

\section{Participantes}

Considerando un muestreo no probabilístico de sujetos voluntarios (Hernández-Sampieri, Fernández-Collado, y Baptista-Lucio, 2014) se obtuvo una muestra por conveniencia de 315 pacientes con asma de un instituto de salud pública de la Ciudad de México, de los cuales 233 (74\%) fueron mujeres y $82(26 \%)$ hombres, con un promedio de edad de $43.96(\mathrm{DE}=14.673$, Rango $=18-75$ años) y de $13.63(\mathrm{DE}$ $=12.401$, Rango = 0.08-61) años padecer la enfermedad. En su mayoría (209) los participantes fueron de la Ciudad de México (66.3\%), 79 del Estado de México (25.1\%) y 27 de otros estados de la República (8.6\%).

Del total, $118(37.5 \%)$ eran casados, 98 (31.1\%) solteros, 45 (14.3\%) vivían en unión libre, 30 (9.5\%) eran separados, 14 (4.4\%) divorciados y 10 (3.2\%) viudos. Respecto al nivel educativo, 80 (25.4\%) tenían licenciatura, $78(24.8 \%)$ secundaria, $68(21.6 \%)$ preparatoria, $41(13 \%)$ carrera técnica, $35(11.1 \%)$ primaria y $13(4.1 \%)$ posgrado. La mayoría (91) se dedicaban al hogar $(28.9 \%), 82$ eran empleados $(26 \%), 36$ profesionistas $(11.4 \%), 36$ comerciantes $(11.4 \%), 27$ estudiantes $(8.6 \%), 26$ desempleados (8.3\%), nueve se dedicaban a algún oficio $(2.9 \%)$ y 8 eran jubilados $(2.5 \%)$.

Los criterios de inclusión necesarios para que los pacientes pudieran participar fueron: 1) ser mayor de edad; 2) diagnóstico confirmado de asma; 3) contar con tratamiento de control; 4) aceptar participar en la investigación, y 5) firma del consentimiento con información. Los criterios de exclusión fueron: 1) tener diagnóstico de asma, pero no haber iniciado tratamiento de control, y 2) además de asma tener diagnóstico de EPOC o bronquiectasias. 


\section{Diseño}

De acuerdo con la clasificación de Ato, López y Benavente (2013) se hizo un estudio de tipo instrumental.

\section{Instrumentos}

Cédula de datos sociodemográficos y clínicos. Conjunto de preguntas para reunir información de datos personales, familiares, educativos, ocupacionales y antecedentes de enfermedad.

Cuestionario Revisado de Percepción de Enfermedad (IPQ-R) (Moss-Morris et al., 2002). Instrumento para la evaluación de la percepción cognitiva y emocional de la enfermedad. La versión original está conformado por 84 ítems divididos en tres áreas diferentes: 1) evaluación de identidad (lista de 14 síntomas experimentados y lista de 14 síntomas percibidos, con formato de respuesta dicotómica sí y no); La puntuación que se usa para evaluar esta parte es la de síntomas percibidos; 2) escalas de control personal, control del tratamiento, temporalidad aguda/crónica, curso cíclico, consecuencias, percepción emocional y coherencia de la enfermedad (conformadas por 38 ítems que evalúan creencias sobre la enfermedad), y 3) escala de causas (conformada por 18 ítems divididas en cuatro áreas: atribuciones de tipo psicológico, inmunidad, casualidad o accidentes y factores de riesgo). La forma de respuesta para la segunda y tercera parte es de tipo Likert de cinco puntos, la cual va de "Totalmente en desacuerdo" a "Totalmente de acuerdo". Cuenta con evidencias de validez estructural, divergente y predictiva, además de datos de estabilidad temporal y consistencia interna alfa de Cronbach de 0.79 a 0.89 para el área 2), y de 0.67 a 0.86 para la escala de causas. Para este estudio se tomó como base la versión en español de Pacheco et al. (2012), modificando el nombre de la enfermedad en las instrucciones y los ítems; se redujeron a cuatro las opciones de respuesta, ajuste aceptado si se consideran las características sociodemográficas, educativas (Díaz-Barriga y González-Celis Rangel, 2019; Lozano, García-Cueto y Muñiz, 2008; Lugo-González et al., 2014) y clínicas de la población (Caldera-Alvarado, Khan, DeFina, Pieper y Brown, 2012), además de prevenir tendencia central de respuesta (DeVellis, 2012; Reyes-Lagunes, 2011).

Cuestionario de Creencias sobre los Medicamentos (BMQ) (Horne, Weinman y Hankins, 1999). Se usó la versión para pacientes mexicanos con asma (Lugo-González y Vega-Valero, 2020), instrumento para evaluar la percepción del tratamiento, el cual está conformado por dos escalas generales: 1) escala de necesidad-preocupación del tratamiento específico para la enfermedad (siete ítems que evalúan las creencias respecto a la necesidad del uso del tratamiento y ocho ítems que evalúan la preocupación por efectos adversos de los mismos -BMQ específico, alfas de Cronbach de 0.83 y 0.79 respectivamente-), y 2) escala de percepción de daño de los medicamentos en general (cinco ítems que evalúan las creencias del efecto dañino que pueden generar - BMQ general, alfa de Cronbach de 0.78-). La forma de respuesta para ambas escalas es de tipo Likert con cuatro puntos, que van de "Totalmente de acuerdo" a "Totalmente en desacuerdo".

Escala de Reporte de Adherencia a la Medicación-Asma (MARS-A) (Horne y Hankins, 2002). Se usó la versión para pacientes mexicanos con asma (Lugo-González y Vega-Valero, 2020). Instrumento unidimensional que evalúa el patrón de conductas de falta de adherencia al medicamento de control. Está constituido por siete ítems que implican conductas intencionales de falta de adherencia y tiene un formato de respuesta tipo Likert de cuatro puntos que van de "Siempre lo hago así" a "Nunca lo hago así". El puntaje de la escala se obtiene sumando las respuestas de cada ítem, por lo que niveles más altos corresponden con un mejor nivel de adherencia y 26 con el punto de corte para diferenciar entre pacientes adherentes de no adherentes. La escala tiene un índice de consistencia interna alfa de Cronbach de 0.85 y con evidencia de validez de constructo con una buena bondad de ajuste (X2 $=20.356, p=0.061$, $\mathrm{X} 2 / \mathrm{gl}=1.69$ ), un buen ajuste absoluto (RMSEA $=0.062$, $\mathrm{GFI}=0.971, \mathrm{AGFI}=0.933)$ y un ajuste comparativo satisfactorio $(\mathrm{TLI}=0.963, \mathrm{NFI}=0.951$ y CFI $=0.97$ ).

Test de Control del Asma (ACT) (Nathan et al., 2004). Instrumento para evaluar el control del asma. Consta de cinco ítems con una escala tipo Likert de frecuencia de cinco puntos. La puntuación máxima es 25, que corresponde con asma controlada; de 20 a 24 puntos corresponde con asma parcialmente controlada, y una puntuación igual o menor a 19 corresponde con asma no controlada. El instrumento tiene evidencia de validez convergente, divergente, de criterio y predictiva, además cuenta con datos de confiabilidad alfa de Cronbach de 0.84 en el índice de consistencia interna alfa.

\section{Análisis de resultados}

Se utilizó el programa estadístico SPSS versión 20 para Windows y se hicieron análisis descriptivos para las características de los pacientes, se evaluó la normalidad de los datos del IPQ-R para determinar el tipo de estadísticos a utilizar en el análisis psicométrico; para esto se usó el estadístico Kolmogorov-Smirnov-Lilliefors (KS) con base en el tamaño de la muestra y el programa utili- 
zado para el análisis de datos (Pedrosa, Juarros, Robles, Basteiro, y García, 2015).

Para efectos del proceso de análisis del IPQ-R, la parte 1) (evaluación de identidad) y la 3) (escala de causas) convergen en la identificación de síntomas atribuibles a la enfermedad y las posibles causas de esta, por lo que sólo se hicieron análisis de tipo descriptivo en formato de lista verificable. Dada esta característica, al menos no se requieren de análisis de tipo psicométrico como confiabilidad (Bot et al., 2003; Corral, 2009).

Respecto a la parte 2) (subescalas de control personal, control del tratamiento, temporalidad aguda/crónica, curso cíclico, consecuencias, percepción emocional y coherencia) se analizó la frecuencia y sesgo para determinar el comportamiento de los ítems; se evaluó la discriminación de reactivos para grupos extremos comparando los cuartiles uno y tres, y la correlación entre ítems y la prueba total, no considerando para futuros análisis a los ítems con correlaciones $<0.20$; se evaluó la confiabilidad con el estadístico alfa de Cronbach (Ferrando y Anguiano, 2010 Reyes-Lagunes y García y Barragán, 2008); después, para evaluar la validez de constructo se hizo un análisis factorial exploratorio con el método de ejes principales con rotación Promax (kappa =4) (Osborne, 2014); para comprobar la pertinencia del modelo factorial se obtuvo el índice $\mathrm{KMO}$, el cual debe ser $\geq 0.8$, y la prueba de esfericidad de Bartlett en la que se debe obtener significancia estadística, en el resultado de $X^{2}$ se determinó el número de factores con base en los autovalores $\geq 1$, se consideró como tres el número de ítems mínimo para conservar un factor y para la agrupación de los ítems a los factores se tomó el criterio de $\geq 0.4$ (Ferrando y Anguiano, 2010; Lloret-Segura, Ferreres-Traver, Hernández-Baeza y Tomás-Marco, 2014).

En seguida se hizo la evaluación de la correlación entre las subdimensiones de la parte dos del IPQ-R, así como la evaluación de la validez convergente y divergente por medio de análisis de correlación entre las subescalas de esta misma parte del IPQ-R, la MARS, el BMQ y el ACT.

\section{Procedimiento}

Los pacientes que participaron en la investigación fueron captados en la sala de espera de su clínica de atención en el instituto de salud; de manera individual a cada paciente se le exponían las razones de ser abordados, se explicaban los objetivos de la investigación y se pedía su participación voluntaria para responder los instrumentos. Cuando los pacientes entregaban los instrumentos se revisaba que estuvieran contestados en su totalidad y se entregaba una copia del consentimiento bajo información.

\section{Resultados}

\section{Evaluación de identidad}

Como se mencionó en la descripción de los instrumentos, la evaluación de identidad está dividida en dos; la primera es acerca de la experiencia de síntomas, y la segunda referente a la identificación de síntomas asociados a la enfermedad. En la figura 1 se muestra la distribución y comparación entre síntomas experimentados (Mediana $=8$, Amplitud Intercuartil $=5$, Rango $=14$ ) y asociados con la enfermedad (Mediana $=9$, Amplitud Intercuartil = 5, Rango $=14$ ). Los síntomas más identificados con la enfermedad fueron dificultad para respirar, silbido en el pecho, tos, flemas (expectoración) y opresión en el pecho, que en sí corresponden con los síntomas definidos por las guías clínicas (GINA, 2019; GUIMA, 2017). En menor frecuencia, pero con un número importante en su reporte, se encuentran cansancio, dificultad para dormir, dolor en el pecho, taquicardia y debilidad, la mayoría de los cuales corresponden con consecuencias de falta de control del asma (GINA, 2019; GUIMA, 2017). Por último, los síntomas poco asociados con la enfermedad fueron irritación en los ojos, mareo, pérdida del apetito sexual y nauseas.

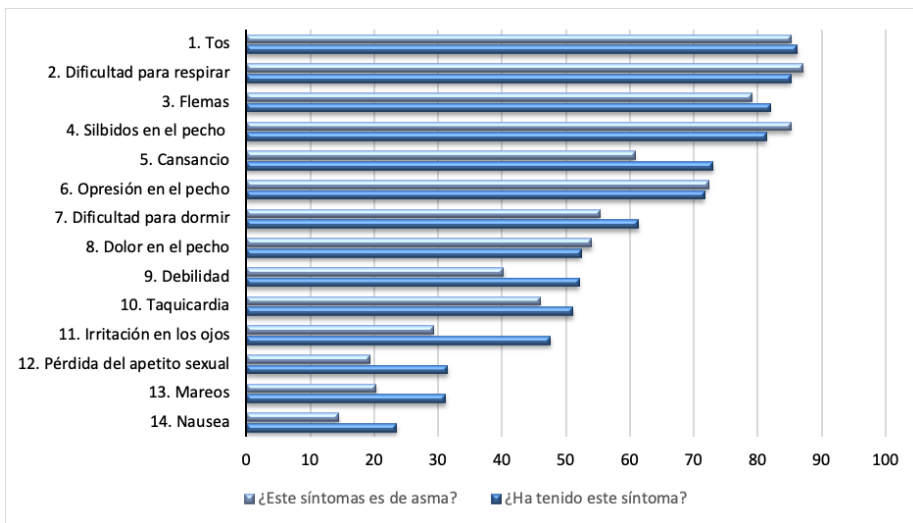

Figura 1.

Síntomas experimentados y síntomas asociados con el asma en la evaluación 1) Identidad del IPQ-R. Resultados obtenidos con una $n=311$.

Además, en esta evaluación se solicita a los pacientes que escriban de uno a tres síntomas que identifiquen con su enfermedad y que no estén mencionados. En este caso algunos pacientes también identificaron acné, ansiedad, ardor en las vías respiratorias, broncoespasmo, calambres, cansancio en los pulmones, constipación nasal, desmayo, dolor pulmonar, dolor de cabeza, dolor en el brazo, escurrimiento nasal, vértigo, urticaria, infla- 
mación estomacal y tristeza, siendo esta última, ansiedad y dolor de cabeza los más reportados.

\section{Grupo de escalas. Escalas de temporalidad crónica-cíclica, consecuencias, control (personal-tratamiento), coherencia y percepción emocional}

En cuanto a su distribución, sesgo, normalidad y correlación, el comportamiento de los ítems del IPQ-R se muestra en la tabla 1 , identificándose que cada una de las opciones de repuesta de los ítems fue elegida. El sesgo, la simetría y el estadístico Kolmogorov-Smirnov-Lilliefors corroboraron que los datos no se comportan de manera normal, justificando el uso de la prueba $U$ de Mann-Whitney para el análisis de discriminación de reactivos para puntajes altos y bajos en temporalidad $(Z=-4.552 \mathrm{a}-10.398, \mathrm{p}<$ $0.01)$, consecuencias $(Z=-3.771 \mathrm{a}-10.067, \mathrm{p}<0.01)$, control personal $(Z=-7.966$ a $-9.736, p<0.01)$, control del tratamiento $(Z=-8.185 \mathrm{a}-9.163, \mathrm{p}<0.01)$, coherencia $(Z=-7.590$ a $-11.346, p<0.01)$, curso cíclico $(Z=$ -9.157 a $-10.686, p<0.01)$ y percepción emocional $(Z$ $=-4.290 \mathrm{a}-10.495, \mathrm{p}<0.01)$.

En la última columna de la tabla 1 (CETC), se observa que el ítem seis de temporalidad (0.173), el 12 de consecuencias (0.053), el 17 y 18 de control personal $(0.127$ y 0.136$)$, y el 33 de percepción emocional (0.100) se encontraron por debajo de 0.20, por lo que no serán considerados para análisis subsecuentes.

\section{Análisis de confiabilidad y validez estructural}

Los resultados en torno a la confiabilidad y validez de la segunda parte del IPQ-R se muestran en la tabla 2, donde los seis factores obtuvieron una consistencia interna aceptable (alfa $=0.874$ a 0.591 ), con excepción del último (control del tratamiento), así como del indicador curso cíclico (alfa $=0.733)$.

La estructura factorial para la segunda parte del IPQ-R cuenta con seis factores (percepción emocional, temporalidad, consecuencias, control personal, coherencia y control del tratamiento) y un indicador (curso cíclico), éstos, con indicadores de adecuación muestral aceptables $(\mathrm{KMO}=0.809, \mathrm{X} 2=3124.389, \mathrm{p}<0.001)$, pasando de 38 a 27 ítems y explicando $51.611 \%$ del total de varianza.

A este último modelo se le aplicaron los siguientes cambios, previos a la solución final.

- Eliminación del ítem 28 de coherencia (0.273) y el ítem 30 de curso cíclico (0.390), dadas sus cargas factoriales.

- Eliminación del ítem 32 de curso cíclico (0.348), dada su carga factorial.
Tabla 1.

Compartimento estadístico de la parte dos del IPQ-R.a

\begin{tabular}{|c|c|c|c|c|c|}
\hline Ítems & $\stackrel{M}{(D E)}$ & $\mathbf{S}$ & C & $\mathrm{KS}_{\mathrm{B}}$ & CETC \\
\hline \multicolumn{6}{|c|}{ TEMPORALIDAD AGUDA/CRÓNICA } \\
\hline $\begin{array}{l}\text { MA durará poco } \\
\text { tiempoc }\end{array}$ & $\begin{array}{l}3.07 \\
(.889)\end{array}$ & -0.667 & -0.353 & 0.229 & 0.476 \\
\hline $\begin{array}{l}\text { Es muy probable } \\
\text { que MA sea para } \\
\text { siempre }\end{array}$ & $\begin{array}{l}2.97 \\
(0.975)\end{array}$ & -0.716 & -0.436 & 0.262 & 0.639 \\
\hline $\begin{array}{l}\text { MA durará } \\
\text { mucho tiempo }\end{array}$ & $\begin{array}{l}2.81 \\
(0.967)\end{array}$ & -0.524 & -0.641 & 0.270 & 0.620 \\
\hline $\begin{array}{l}\text { MA se me } \\
\text { quitará prontoc }\end{array}$ & $\begin{array}{l}2.58 \\
(1.36)\end{array}$ & 97 & -0.311 & 0.250 & 0.570 \\
\hline $\begin{array}{l}\text { Pienso que } \\
\text { tendré } A \text { el resto } \\
\text { de mi vida }\end{array}$ & $\begin{array}{l}2.96 \\
(0.965)\end{array}$ & -0.684 & -0.452 & 0.258 & 0.593 \\
\hline $\begin{array}{l}\text { MA mejorará con } \\
\text { el tiempo }\end{array}$ & $\begin{array}{l}2.39 \\
(1.06)\end{array}$ & -0.709 & -0.043 & 0.294 & 0.173 \\
\hline \multicolumn{6}{|c|}{ Consecuencias } \\
\hline $\begin{array}{l}\text { MA causa } \\
\text { dificultades a los } \\
\text { que me rodean }\end{array}$ & $\begin{array}{l}2.39 \\
(1.06)\end{array}$ & 0.160 & -1.19 & 0.201 & 0.560 \\
\hline $\begin{array}{l}\text { MA tiene serias } \\
\text { consecuencias } \\
\text { económicas }\end{array}$ & $\begin{array}{l}2.95 \\
(0.907)\end{array}$ & -0.559 & -0.463 & 0.247 & 0.547 \\
\hline $\begin{array}{l}\text { MA es una } \\
\text { enfermedad } \\
\text { grave }\end{array}$ & $\begin{array}{l}2.81 \\
(0.906)\end{array}$ & -0.350 & -0.659 & 0.241 & 0.526 \\
\hline $\begin{array}{l}\text { MA afecta } \\
\text { mucho la manera } \\
\text { en la que me ven } \\
\text { los demás }\end{array}$ & $\begin{array}{l}2.11 \\
(0.968)\end{array}$ & 0.496 & -0.734 & 0.231 & 0.470 \\
\hline $\begin{array}{l}\text { MA ocasiona } \\
\text { consecuencias } \\
\text { importantes en } \\
\text { mi vida }\end{array}$ & $\begin{array}{l}2.72 \\
(0.953)\end{array}$ & -0.338 & -0.782 & 0.248 & 0.553 \\
\hline $\begin{array}{l}\text { MA no repercute } \\
\text { mucho en mi } \\
\text { vidac }\end{array}$ & $\begin{array}{l}2.60 \\
(0.928)\end{array}$ & -0.011 & -0.880 & 0.216 & 0.053 \\
\hline
\end{tabular}

\section{Control personal}

Tengo la

capacidad para

controlar MA

3.09
$(0.813)$

$-0.755 \quad 0.254$

0.270

0.290

Los cambios en los síntomas de

MA dependen

2.98

$(0.855)$

$-0.494 \quad-0.219 \quad 0.245$

0.287

de $\mathrm{mi}$

a MI: mi asma, A: asma, S: Simetría; C: Curtosis; KS:

Estadístico Kolmogorov-Smirnov-Lilliefors; CETC: Correlación elemento-total corregida. ${ }^{\mathrm{b}} \mathrm{p}<0.01$. c Ítems inversos. 


\begin{tabular}{|c|c|c|c|c|c|}
\hline Ítems & $\underset{\text { (DE) }}{M}$ & $\mathbf{S}$ & C & $\mathrm{KS}_{\mathrm{B}}$ & CETC \\
\hline $\begin{array}{l}\text { Lo que yo haga } \\
\text { puede hacer que } \\
\text { MA mejore o } \\
\text { empeore }\end{array}$ & $\begin{array}{l}2.08 \\
(0.995)\end{array}$ & -0.494 & -0.423 & 0.257 & 0.445 \\
\hline $\begin{array}{l}\text { Hay muchas } \\
\text { cosas que puedo } \\
\text { hacer para } \\
\text { controlar los } \\
\text { síntomas de MA }\end{array}$ & $\begin{array}{l}3.32 \\
(0.667)\end{array}$ & -0.931 & 1.54 & 0.274 & 0.470 \\
\hline $\begin{array}{l}\text { Cualquier cosa } \\
\text { que yo haga no } \\
\text { ocasiona cambios } \\
\text { en el control de } \\
\text { MAc }\end{array}$ & $\begin{array}{l}2.75 \\
(0.911)\end{array}$ & -0.282 & -0.712 & 0.238 & 0.127 \\
\hline $\begin{array}{l}\text { Mis acciones no } \\
\text { tienen efecto } \\
\text { en el control de } \\
\text { MAc }\end{array}$ & $\begin{array}{l}2.82 \\
(0.903)\end{array}$ & -0.432 & -0.538 & 0.260 & 0.139 \\
\hline
\end{tabular}

\section{Control del tratamiento}

El tratamiento que me dan los médicos ayuda a $\begin{array}{lllll}3.45 & 0.487 & -1.26 & 0.315 & 0.426\end{array}$ controlar MA

Mi tratamiento será efectivo para controlar MA
Las

consecuencias

de tener $A$

se pueden

prevenir con el

tratamiento

Hay poco que se pueda hacerse

para controlar

MAc

$$
\begin{array}{lllll}
3.15 & 0.734 & 0.399 & 0.267 & 0.365 \\
(0.764) & -0.734
\end{array}
$$

No hay nada que pueda ayudar a controlar MAc

$\begin{array}{lllll}3.14 & -0.861 & 0.547 & 0.273 & 0.308\end{array}$

$\begin{array}{lllll}3.31 & -1.20 & 1.34 & 0.272 & 0.307 \\ (0.788) & & & & \end{array}$

\section{Coherencia}

\begin{tabular}{|c|c|c|c|c|c|}
\hline $\begin{array}{l}\text { Entiendo } \\
\text { claramente MA }\end{array}$ & $\begin{array}{l}3.03 \\
(0.804)\end{array}$ & -0.611 & 0.043 & 0.274 & 0.376 \\
\hline $\begin{array}{l}\text { Los síntomas de } \\
\text { MA son difíciles } \\
\text { de entenderc }\end{array}$ & $\begin{array}{l}2.92 \\
(0.865)\end{array}$ & -0.539 & -0.281 & 0.269 & 0.563 \\
\hline
\end{tabular}

a MI: mi asma, A: asma, S: Simetría; C: Curtosis; KS:

Estadístico Kolmogorov-Smirnov-Lilliefors; CETC: Correlación elemento-total corregida. ${ }^{\mathrm{b}} \mathrm{p}<0.01$. c Ítems inversos.

\begin{tabular}{llllll}
\multicolumn{1}{c}{ Ítems } & \multicolumn{1}{c}{$\begin{array}{c}\text { M } \\
\text { (DE) }\end{array}$} & S & C & KS & CETC \\
\hline $\begin{array}{l}\text { Hay muchas } \\
\text { cosas que } \\
\text { desconozco de } \\
\text { MAc }\end{array}$ & $\begin{array}{l}2.61 \\
(0.937)\end{array}$ & -0.011 & -0.915 & 0.218 & 0.556 \\
$\begin{array}{lllll}\text { No entiendo } \\
\text { MA R }\end{array}$ & $\begin{array}{l}2.84 \\
(0.902)\end{array}$ & -0.403 & -0.595 & 0.247 & 0.636 \\
$\begin{array}{l}\text { MA no tiene } \\
\text { ningún sentido } \\
\text { para míc }\end{array}$ & $\begin{array}{l}3.09 \\
(0.866)\end{array}$ & -0.748 & -0.063 & 0.249 & 0.388 \\
\hline
\end{tabular}

\section{Curso cíclico}

\begin{tabular}{|c|c|c|c|c|c|}
\hline $\begin{array}{l}\text { MA mejora y } \\
\text { empeora por } \\
\text { temporadas }\end{array}$ & $\begin{array}{l}3.22 \\
(0.752)\end{array}$ & -0.977 & 1.16 & 0.274 & 0.513 \\
\hline $\begin{array}{l}\text { MA es muy } \\
\text { impredecible }\end{array}$ & $\begin{array}{l}2.66 \\
(0.903)\end{array}$ & -0.254 & -0.680 & 0.251 & 0.491 \\
\hline $\begin{array}{l}\text { Los síntomas de } \\
\text { MA van y vienen } \\
\text { por temporadas }\end{array}$ & $\begin{array}{l}3.10 \\
(0.830)\end{array}$ & -0.880 & 0.495 & 0.283 & 0.525 \\
\hline $\begin{array}{l}\text { Los síntomas } \\
\text { de A cambian } \\
\text { mucho de un día } \\
\text { para otro }\end{array}$ & $\begin{array}{l}2.55 \\
(0.970)\end{array}$ & -0.016 & -0.976 & 0.203 & 0.519 \\
\hline
\end{tabular}
para otro

\section{Percepción emocional}

\begin{tabular}{|c|c|c|c|c|c|}
\hline $\begin{array}{l}\text { MA no me } \\
\text { preocupac }\end{array}$ & $\begin{array}{l}2.92 \\
(0.998)\end{array}$ & -0.617 & -0.656 & 0.250 & 0.100 \\
\hline $\begin{array}{l}\text { Tener A me hace } \\
\text { sentir ansioso(a) }\end{array}$ & $\begin{array}{l}2.48 \\
(0.978)\end{array}$ & 0.074 & -0.987 & 0.213 & 0.594 \\
\hline $\begin{array}{l}\text { Me deprimo } \\
\text { cuando pienso en } \\
\text { mi MA }\end{array}$ & $\begin{array}{l}2.26 \\
(1.03)\end{array}$ & 0.267 & -1.10 & 0.196 & 0.687 \\
\hline $\begin{array}{l}\text { MA hace que } \\
\text { me sienta } \\
\text { temeroso(a) }\end{array}$ & $\begin{array}{l}2.27 \\
(0.998)\end{array}$ & 0.234 & -1.01 & 0.202 & 0.684 \\
\hline $\begin{array}{l}\text { MA me hace } \\
\text { sentir enojado(a) }\end{array}$ & $\begin{array}{l}2.19 \\
(0.981)\end{array}$ & 0.363 & -0.894 & 0.218 & 0.709 \\
\hline $\begin{array}{l}\text { Me preocupo } \\
\text { cuando pienso } \\
\text { en MA }\end{array}$ & $\begin{array}{l}2.51 \\
(1.00)\end{array}$ & -0.153 & -1.05 & 0.238 & 0.716 \\
\hline
\end{tabular}

a MI: mi asma, A: asma, S: Simetría; C: Curtosis; KS:

Estadístico Kolmogorov-Smirnov-Lilliefors; CETC: Correlación elemento-total corregida. ${ }^{\mathrm{b}} \mathrm{p}<0.01$. c Ítems inversos.

- Eliminación del ítem 24 de coherencia, porque se agrupó en la subescala de control del tratamiento y no es congruente con el constructo.

- Eliminación de los ítems 22 (0.335) y 23 (0.377) de control del tratamiento, dadas sus cargas factoriales. 
Artículo Empírico | Propiedades psicométricas...| Lugo-González, Fernández-Vega, Pérez-Bautista \&Z Vega-Valero

Tabla 2.

Estructura factorial del IPQ-R para pacientes mexicanos con asma.a

\begin{tabular}{|c|c|c|c|c|c|c|c|c|}
\hline \multirow{2}{*}{ ÍTEMS } & \multicolumn{7}{|c|}{ FACTORES } & \multirow{2}{*}{$A$} \\
\hline & 1 & 2 & 3 & 4 & 5 & 6 & 7 & \\
\hline
\end{tabular}

Percepción emocional

\begin{tabular}{|c|c|c|c|c|c|c|c|c|}
\hline MA hace que me sienta temeroso(a) & 0.852 & -0.018 & -0.079 & -0.044 & -0.002 & -0.031 & 0.108 & 0.874 \\
\hline Me preocupo cuando pienso en MA & 0.808 & 0.089 & -0.029 & -0.035 & -0.039 & -0.032 & 0.073 & \\
\hline Me deprimo cuando pienso en MA & 0.768 & -0.001 & 0.039 & -0.042 & 0.075 & 0.012 & -0.061 & \\
\hline MA me hace sentir enojado(a) & 0.744 & -0.010 & 0.081 & 0.022 & -0.048 & -0.027 & -0.018 & \\
\hline Tener A me hace sentir ansioso(a) & 0.593 & -0.038 & 0.068 & -0.016 & 0.095 & 0.212 & -0.068 & \\
\hline \multicolumn{9}{|c|}{ TEMPORALIDAD AGUDA/CRÓNICA } \\
\hline Es muy probable que MA sea para siempre & 0.028 & 0.808 & -0.005 & 0.176 & -0.126 & -0.060 & -0.029 & 0.808 \\
\hline MA durará mucho tiempo & 0.067 & 0.800 & -0.070 & 0.163 & -0.141 & -0.053 & -0.054 & \\
\hline Pienso que tendré $\mathrm{A}$ el resto de mi vida & -0.028 & 0.701 & 0.104 & 0.060 & 0.001 & 0.013 & -0.037 & \\
\hline MA se me quitará prontob & -0.067 & 0.576 & 0.009 & -0.252 & 0.151 & 0.028 & 0.035 & \\
\hline MA durará poco tiempob & 0.009 & 0.518 & -0.054 & -0.283 & 0.152 & 0.091 & 0.070 & \\
\hline \multicolumn{9}{|c|}{ Consecuencias } \\
\hline MA tiene graves consecuencias económicas & -0.087 & -0.026 & 0.751 & -0.043 & 0.015 & -0.041 & 0.098 & 0.779 \\
\hline MA causa dificultades a los que me rodean & 0.033 & -0.008 & 0.659 & 0.088 & 0.020 & 0.072 & -0.176 & \\
\hline MA ocasiona consecuencias importantes en mi vida & 0.169 & 0.020 & 0.601 & 0.009 & 0.019 & -.088 & .124 & \\
\hline MA es una enfermedad grave & 0.029 & 0.092 & 0.573 & -0.084 & -0.012 & 0.016 & 0.105 & \\
\hline $\begin{array}{l}\text { MA afecta mucho la manera en la que me ven los } \\
\text { demás }\end{array}$ & 0.230 & -0.118 & 0.442 & 0.055 & -0.114 & -0.059 & -0.155 & \\
\hline
\end{tabular}

\section{Control personal}

Hay cosas que puedo hacer para controlar los síntomas de MA

$\begin{array}{llllllll}-0.084 & 0.020 & 0.076 & 0.719 & -0.007 & 0.072 & 0.094 & 0.739\end{array}$

Los cambios en los síntomas de MA dependen de mí

$\begin{array}{lllllll}0.032 & 0.022 & -0.166 & 0.598 & 0.127 & -0.043 & -0.022\end{array}$

Tengo la capacidad para controlar MA

$\begin{array}{lllllll}-0.039 & -0.034 & -0.023 & 0.581 & 0.025 & -0.040 & 0.009\end{array}$

Lo que yo haga puede hacer que MA mejore o empeore

$\begin{array}{lllllll}-0.024 & 0.044 & 0.095 & 0.573 & 0.128 & 0.117 & 0.078\end{array}$

\section{Coherencia}

$\begin{array}{lllllllll}\text { Hay muchas que desconozco acerca de MAb } & 0.013 & -0.063 & 0.030 & 0.067 & 0.813 & -0.037 & -0.073 & 0.777 \\ \text { No entiendo MAb } & 0.027 & 0.000 & 0.022 & 0.081 & 0.742 & -0.060 & -0.042 \\ \text { Los síntomas de MA son difíciles de entenderb } & 0.009 & 0.012 & -0.054 & 0.077 & 0.616 & -0.009 & 0.098\end{array}$

Control del tratamiento

El tratamiento que me dan los médicos ayuda a controlar MA

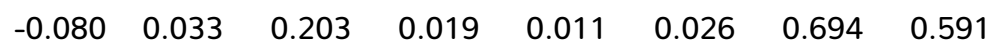
Las consecuencias de tener A se pueden prevenir con el tratamiento

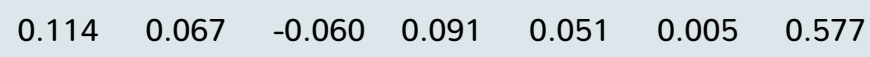

a Análisis exploratorio efectuado con el método de ejes principales y rotación Promax, Kappa = 4 (Osborne, 2014); MA: mi asma: A: asma; KMO: Índice Kaiser-Meyer-Olkin; VTE: Varianza total explicada. ${ }^{b}$ Ítems inversos. c $\mathrm{p}<0.001$. 


\begin{tabular}{|c|c|c|c|c|c|c|c|c|}
\hline \multirow{2}{*}{ Ítems } & \multicolumn{7}{|c|}{ FACTORES } & \multirow{2}{*}{$A$} \\
\hline & 1 & 2 & 3 & 4 & 5 & 6 & 7 & \\
\hline Mi tratamiento será efectivo para controlar MA & 0.034 & -0.264 & -0.079 & 0.062 & -0.147 & -0.030 & 0.486 & \\
\hline \multicolumn{9}{|c|}{ INDICADOR } \\
\hline \multicolumn{9}{|c|}{ Curso cíclico } \\
\hline MA mejora y empeora por temporadas & -0.062 & 0.037 & 0.091 & -0.048 & -0.059 & 0.814 & -0.021 & 0.733 \\
\hline \multirow[t]{4}{*}{ Los síntomas de A van y vienen por temporadas } & 0.120 & -0.057 & -0.152 & 0.128 & -0.048 & 0.715 & 0.036 & \\
\hline & & & & & & & $\mathrm{KMO}$ & 0.809 \\
\hline & & & & & & & $x^{2}$ & B24.389c \\
\hline & & & & & & & VTE & 51.611 \\
\hline
\end{tabular}

a Análisis exploratorio efectuado con el método de ejes principales y rotación Promax, Kappa = 4 (Osborne, 2014); MA: mi asma: A: asma; KMO: Índice Kaiser-Meyer-Olkin; VTE: Varianza total explicada. ${ }^{b}$ Ítems inversos. c p $<0.001$.

\section{Correlación entre factores}

En la tabla 3 se muestra que las correlaciones fuertes se presentaron entre las dos subdimensiones de síntomas (experiencia-identidad), consecuencias y percepción emocional de manera positiva. Las correlaciones moderadas se observaron entre síntomas (experiencia-identidad), consecuencias y percepción emocional, control personal y control del

Tabla 3.

Correlación entre subdimensiones de la parte dos del IPQ-R. del tratamiento y necesidad (bajo), y percepción emocional y necesidad (moderado). Para la validez divergente se analizó la relación entre consecuencias y control del asma (moderado), coherencia y preocupación (moderado); percepción emocional y control del asma (moderado), e identidad y control del asma (moderada).

\section{Escala de causas}

\begin{tabular}{|c|c|c|c|c|c|c|c|c|}
\hline SUBdimENSIONES & 1 & 2 & 3 & 4 & 5 & 6 & 7 & 8 \\
\hline \multicolumn{9}{|l|}{ 1. Experiencia síntomas } \\
\hline 2. Identidad & $0.706^{b}$ & & & & & & & \\
\hline 3. Temporalidad & 0.026 & 0.044 & & & & & & \\
\hline 4. Consecuencias & $0.304^{b}$ & $0.368^{b}$ & $0.191^{\mathrm{b}}$ & & & & & \\
\hline 5. Control personal & $-0.189^{b}$ & $-0.190^{b}$ & 0.107 & -0.033 & & & & \\
\hline 6. Control tratamiento & $-0.137^{a}$ & $-0.118^{a}$ & -0.001 & 0.023 & $0.367^{b}$ & & & \\
\hline 7. Coherencia & -0.011 & -0.057 & $0.124^{\mathrm{a}}$ & $-0.222^{b}$ & $0.257^{b}$ & 0.109 & & \\
\hline 8. Curso cíclico & $0.121^{a}$ & $0.182^{b}$ & 0.082 & $0.243^{b}$ & $0.186^{b}$ & $0.227^{b}$ & -0.037 & \\
\hline $\begin{array}{l}\text { 9. Percepción emocional } \\
{ }^{a} p<0.05 \text {. }^{b} p<0.01 \text {. }\end{array}$ & $0.327^{b}$ & $0.382^{\mathrm{b}}$ & 0.061 & $0.560^{\mathrm{b}}$ & -0.091 & 0.028 & $-0.258^{b}$ & $0.266^{b}$ \\
\hline
\end{tabular}

tratamiento, y curso cíclico y percepción emocional.

\section{Validez convergente y divergente}

Para la validez convergente se analizaron diversas relaciones teóricamente justificadas y su grado de asociación (tabla 4), como temporalidad, adherencia y necesidad (bajo), consecuencias y necesidad (moderado), control personal y control del asma (moderado), control
La manera de responder esta escala originalmente es en términos de un continuo en desacuerdo a de acuerdo, lo que según la versión original posibilita factorizar las causas asociadas a la enfermedad. Sin embargo, ya que lo que es necesario identificar es lo que los pacientes creen que causó su enfermedad, se agruparon las respuestas "De acuerdo" y "Totalmente de acuerdo" en la categoría "Sí", y "En desacuerdo" y "Totalmente en desacuerdo" en "No". 
Tabla 4.

Nivel de correlación entre subdimensiones de la parte dos del IPQ-R, MARS, BMQ y ACT. ${ }^{a}$

\begin{tabular}{|c|c|c|c|c|}
\hline INSTRUMENTO & MARS & \multicolumn{2}{|c|}{ BMQ } & \multirow{2}{*}{$\begin{array}{c}\text { ACT } \\
\text { CONTROL } \\
\text { DEL ASMA }\end{array}$} \\
\hline SUBdiMENSIÓN & AdHerencia & Necesidad & Preocupación & \\
\hline \multicolumn{2}{|l|}{$\begin{array}{l}\text { Experiencia } \\
\text { síntomas }\end{array}$} & $0.143^{b}$ & $0.248^{c}$ & $-0.370^{c}$ \\
\hline \multicolumn{2}{|l|}{ Identidad } & $0.189^{c}$ & $0.254^{c}$ & $-0.419^{c}$ \\
\hline Temporalidad & $0.173^{c}$ & $0.233^{c}$ & & \\
\hline \multicolumn{2}{|l|}{ Consecuencias } & $0.418^{c}$ & $0.437^{c}$ & $-0.326^{c}$ \\
\hline \multicolumn{2}{|l|}{$\begin{array}{l}\text { Control } \\
\text { personal }\end{array}$} & $0.150^{c}$ & -0.105 & $0.319^{c}$ \\
\hline \multicolumn{2}{|l|}{$\begin{array}{l}\text { Control } \\
\text { tratamiento }\end{array}$} & $0.255^{c}$ & & $0.127^{b}$ \\
\hline \multicolumn{2}{|l|}{ Coherencia } & & $-0.420^{c}$ & $0.181^{c}$ \\
\hline \multicolumn{2}{|l|}{ Curso cíclico } & $0.156^{c}$ & $0.187^{c}$ & $-0.198^{c}$ \\
\hline \multicolumn{2}{|l|}{$\begin{array}{l}\text { Percepción } \\
\text { emocional }\end{array}$} & $0.290^{c}$ & $0.512^{c}$ & $-0.419^{c}$ \\
\hline \multicolumn{5}{|c|}{$\begin{array}{l}\text { Sólo se incluyeron las correlaciones moderadas y altas. }{ }^{*} p \\
<.05,{ }^{* *} p<.01 .\end{array}$} \\
\hline
\end{tabular}

En la figura 2 se muestran las principales causas asociadas con el asma (en este caso contaminación ambiental, alteración de las defensas del cuerpo, estrés o preocupaciones, atención medica deficiente en el pasado y el estado emocional - tristeza o ansiedad — fueron las más frecuentes). Respecto a las causas menos frecuentes destacaron accidentes, consumo de alcohol, pensar de manera negativa, personalidad, casualidad y la mala suerte.

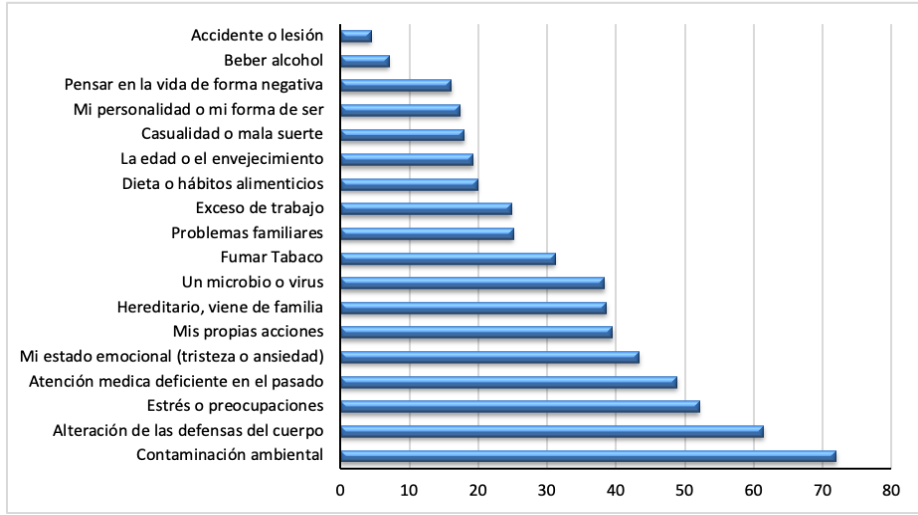

Figura 2.

Descripción de causas asociadas con el asma.

Como en el caso de la evaluación de identidad, también se les indica que ordenen en importancia las tres primeras causas de su enfermedad; en este caso lo que se encontró fue: contaminación (exposición al humo de tabaco y leña), estrés, estados emocionales ne- gativos y la presencia de enfermedades respiratorias en la niñez, su cuidado inadecuado y mal tratamiento.

\section{Interpretación de las puntuaciones del IPQ-R}

En la subescala de identidad se identificará el tipo y número de síntomas identificados con el asma y a mayor número de síntomas, mayor identidad. En la subescala de causas se obtendrá información respecto a las causas asociadas al desarrollo de asma. Por último, a mayor puntuación en representación emocional, temporalidad, consecuencias, control personal, coherencia, curso cíclico y control del tratamiento, mayor será la percepción de impacto emocional, cronicidad de la enfermedad, repercusiones negativas, autoeficacia para el control del asma, entendimiento de la enfermedad, ciclicidad de la enfermedad y sus síntomas y efectos positivos del tratamiento, respectivamente.

\section{Discusión}

Ya que el objetivo de este trabajo fue evaluar las propiedades psicométricas del IPQ-R en adultos con asma, se puede concluir que el instrumento cuenta con propiedades psicométricas adecuadas para la evaluación de la percepción de enfermedad en este tipo de pacientes; sin embargo, se sugiere tomar con cautela los resultados del factor control del tratamiento porque tiene datos de confiabilidad bajos (alfa $=0.591$ ). Los resultados con relación a la escala de identidad mostraron la necesidad de incluir síntomas asociados a la enfermedad, como dolor de cabeza, ansiedad y tristeza, ya que fueron los que se reportaron de manera más consistente en el apartado del instrumento donde se les solicitaba colocar síntomas asociados con el asma que no estuvieran mencionados.

Ahora bien, se sabe que los principales síntomas de asma son la dificultad respiratoria, opresión en el pecho, sibilancias, flemas y tos (GINA, 2016; GUIMA, 2017), siendo identificados sin problema por los pacientes; sin embargo, se observa que la mayoría de los pacientes identifican como síntomas las consecuencias físicas, funcionales y psicológicas derivadas del control inadecuado del asma, como el cansancio o debilidad, dificultad para dormir, tristeza y ansiedad, entre otros (GINA, 2016; GUIMA, 2017).

En virtud de los resultados en la evaluación de identidad, donde experiencia de síntomas e identidad se correlacionan de manera muy importante (Rho $=0.706$ ), se considera innecesario que se utilicen ambas listas de síntomas (es la misma lista); además, la lista de experiencia de síntomas carece de instrucción temporal y, para evaluar la subdimensión de ese apartado, sólo se usa la se- 
gunda lista (Moss-Morris et al., 2002). En contraparte, es necesario comentar que la versión adaptada por Pacheco et al. (2012) propone que en la escala de identidad se incluya una tercera forma de respuesta: identificar si los síntomas se asocian al tratamiento médico. Por su parte, en la versión de Hurtado-Piña (2017) la lista de síntomas fue adaptada para pacientes con obesidad, y la opción de respuesta incluye tres posibles formas: "No tengo este padecimiento", "Sí tengo este padecimiento, pero no está relacionado con mi sobrepeso" y "Tengo este padecimiento y si está relacionado con mi sobrepeso".

Respecto a la sección 2 del IPQ-R, en general se puede mencionar que se replicó la estructura factorial propuesta en la versión original (Moss-Morris et al., 2002), así como en la versión para enfermedades cardiacas (Brink et al., 2011), hipertensión (Beléndez et al., 2005), cáncer (Giannousi et al., 2010; Moon et al., 2017), dermatitis atópica (Wittkowski et al., 2008) y fibromialgia (Van Ittersum et al., 2009), aunque sólo con 27 ítems y un factor pasando a ser indicador.

Con relación a los datos de confiabilidad para los factores de control personal $\alpha=0.739$, consecuencias $\alpha=0.779$, temporalidad $\alpha=0.808$ y percepción emocional $\alpha=0.874$, hay algunas discrepancias con el IPQ-R original (Moss-Morris et al., 2002), en las subescalas de control personal $\alpha=0.81$, consecuencias $\alpha=0.84$ y temporalidad $\alpha=0.89$; a pesar de ello los datos de este estudio son aceptables. Respecto a la versión mexicana para pacientes con obesidad (Hurtado-Piña, 2017), los datos son similares en percepción emocional $\alpha=0.85$, en la agrupación de control y consecuencias $\alpha=0.74$, pero no en temporalidad $\alpha=0.73$.

En lo que respecta a la evaluación de la validez estructural es necesario indicar que dos de las versiones en español y la versión original (Beléndez et al., 2005; Hurtado-Piña, 2017; Moss-Morris et al., 2002) utilizaron técnicas de factorización basados en el análisis de componentes principales con rotación varimax, lo cual es considerado inadecuado porque este tipo de factorizaciones no se enfocan a identificar una estructura factorial, sino a reducir el número de variables. Por ello se optó por usar el método de ejes principales, además de que se recomienda para datos que no se comportan de manera normal (Osborne, 2014).

En este estudio se eliminaron 11 ítems de la segunda parte del IPQ-R, seis de ellos fueron ítems inversos, lo que podría sugerir que hubo dificultad para entender su contenido o que no aplican en el contexto de la enfermedad y con la perspectiva de los pacientes (por ejemplo, el ítem seis de temporalidad — Mi asma mejorará con el tiempo" —, el cual no implica una descripción del asma como enfermedad crónica, aguda o cíclica, el ítem 32 de curso cíclico - "Los síntomas de asma cambian mucho de un día para otro" —, si bien el asma se caracteriza por periodos sintomáticos y asintomáticos (GINA, 2019; GUIMA, 2017) no necesariamente el cambio es de un día para otro, y los ítems 22 y 23 de control del tratamiento, que describen que hay poco y nada que se pueda hacer para controlar el asma, no indican algún tratamiento.

Ahora bien, con base en los resultados obtenidos en los datos de validez convergente se identificó que una percepción de temporalidad crónica del asma está asociada con la necesidad del tratamiento y adherencia al tratamiento de control, una percepción elevada de consecuencias se asocia con una mayor necesidad del tratamiento de control, datos similares propuestos por Horne y Weinman (2002); sin embargo, es importante que esta última asociación también se da con la preocupación por efectos adversos del tratamiento, lo que indica dos formas de valorar la relación de las consecuencias con la percepción del tratamiento.

Si se perciben elevadas consecuencias de la enfermedad se tenderá, por un lado, a percibir que el tratamiento es muy necesario, o se considerará que el tratamiento puede generar consecuencias o es inefectivo, datos que además incluyen los aspectos emocionales (percepción emocional) y que concuerdan con las evidencias de Bender y Bender (2005) y Unni y Shiyanbola (2016). Respecto al control personal, el vínculo directo es con el control del asma, comprobando que la percepción de control de la enfermedad no necesariamente está asociada con la percepción de control conductual; en este caso con las conductas de adherencia al tratamiento de control (Ajzen y Fishbein, 1980).

Referente a la validez divergente se identificó que una elevada percepción de consecuencias se asocia de modo negativo con el control del asma y preocupación, justificado esto porque, al percibir más consecuencias, seguramente se experimentan más síntomas, más consecuencias emocionales y funcionales derivadas del mal control del asma (GINA, 2019; GUIMA, 2017; Halm et al., 2006; Unni y Shiyanbola, 2016).

Estos resultados tienen varias lecturas; por un lado la falta de asociación entre las creencias acerca del asma y adherencia se ha descrito en diferentes estudios de revisión y metanálisis en los que se ha cuestionado no sólo la función de las creencias en cuanto a la enfermedad como variables asociadas a la adherencia, sino del MSCRE (Aujla et al., 2016; Brandes y Mullan, 2014; Holmes, Hughes y Morrison, 2014; Kucukarslan, 2012). Por otro lado, las criticas vienen desde el mismo 
MSCRE, esto es, se considera que la evaluación de las subdimensiones del MSCRE enfocadas en el instrumento (Moss-Morris et al., 2002) tienen limitaciones porque los ítems son genéricos y no son ítems diseñados con base en el proceso del MSCRE, no son dirigidos a una enfermedad específica y no efectúan un análisis individualizado de la percepción de la enfermedad (Leventhal et al., 2010; Mora y McAndrew, 2013); ahora, esto no implica que el IPQ-R no evalúe la percepción de enfermedad; lo que no evalúa es el proceso del modelo.

A pesar de lo anterior, las investigaciones proponen que una de las posibles causas de la falta de asociación entre estas variables es que las creencias acerca del asma se enfocan a la enfermedad y no a la conducta de adherencia, por lo que las creencias más cercanas a la adherencia serían las del tratamiento, no de la enfermedad (Ajzen y Fishbein, 1980). Horne y Weinman (2002) propusieron un modelo en el que las creencias acerca del tratamiento mediaban las creencias respecto a la enfermedad y las conductas de adherencia; dicho modelo fue probado de modo experimental y arrojó resultados que corroboran esta hipótesis (Kucukarslan, Lee, Patel y Ruperalia, 2013; Kucukarslan, Plumley, Chang y Ueda, 2014). De hecho, Kucukarslan (2012), Aujla et al. (2016) y Unni y Shiyanbola (2016) consideran necesario incluir las creencias acerca del tratamiento, desde el modelo Horne et al. (1999) al MSCRE, para el trabajo de explicación, predicción e intervención en conducta de adherencia en personas con asma y otras enfermedades crónicas.

Respecto a la escala de causas el proceso de ajuste de respuestas, eliminación e inclusión de causas se dio del mismo modo que en la escala de identidad. El IPQ-R (Moss-Morris et al., 2002) utiliza en esta escala una opción de respuesta tipo Likert, lo cual implica estar en cierto nivel de acuerdo o en desacuerdo con una posible causa; además, con esta manera de respuesta es posible obtener datos de confiablidad y la estructura factorial de las causas; sin embargo, al finalizar la escala se les pregunta acerca de las tres causas más importantes para que desarrollaran la enfermedad; caso similar sucede en el BIPQ (Broadbent et al., 2006); entonces la pregunta es si se desea saber qué es lo que creen que causó su enfermedad o con qué causas están más de acuerdo. Dado este contexto, se determinó que la opción de respuesta fuera "Sí, sí creo que causó mi enfermedad" o "No, no creo que causó mi enfermedad", ya que así se identifica cuáles son las causas atribuidas al desarrollo de su enfermedad; además se identificó que los pacientes confunden en diferenciar entre causa de enfermedad y causa de síntomas de enfermedad. Es necesario mencionar que la forma de respuesta que se sugiere es similar con la primera versión del IPQ (Weinman et al., 1996).

Por último, las opciones de causas de enfermedad, como beber alcohol y accidente o lesión, fueron eliminadas debido a su baja frecuencia; fumar y estar expuesto al humo de tabaco se incluyó en una misma causa; además se incluyeron como causas usar o exponerse al humo de leña o petróleo, cloro, químicos, frío, humedad y enfermedades respiratorias mal atendidas; esto con base en las respuestas proporcionadas por los pacientes en la pregunta de las tres principales causas asociadas a su enfermedad.

\section{Conclusiones}

Con base en las evidencias encontradas se concluye que el IPQ-R es un instrumento confiable y válido para la evaluación de la percepción de enfermedad en pacientes con asma. Es importante mencionar que la forma de obtención de la muestra puede representar un problema porque fue de tipo no probabilístico, y la mayoría de los participantes fueron residentes de la Ciudad de México.

Se considera necesario continuar acumulando evidencias como la estabilidad temporal, validez de tipo predictivo y estudios de tipo confirmatorio. Además, derivado de los resultados de validez convergente y divergente, sería importante probar modelos en los que se identifique la tendencia de asociación entre creencias de enfermedad y del tratamiento, para así explicar de manera más robusta las conductas de adherencia y el control de la enfermedad.

\section{Referencias}

Aberkane, S. (2017). Psychometric testing of an Arabic translation of the Revised-IIIness Perception Questionnaire (IPQ-R) for chronic illness patients. The Malaysian Journal of Medical Sciences, 24(4), 74-85. https://doi.org/10.21315/ mjms2017.24.4.9

Abubakari, A. R., Jones, M. C., Lauder, W., Kirk, A., Devendra, D., \& Anderson, J. (2012). Psychometric properties of the Revised Illness Perception Questionnaire: Factor Structure and Reliability among African-Origin Populations with Type 2 Diabetes. International Journal of Nursing Studies, 49(6), 672-681. doi:10.1016/j.ijnurstu.2011.11.008

Achstetter, L. I., Schultz, K., Faller, H., \& Schuler, M. (2016). Leventhal's common-sense model and asthma control: Do illness representations predict success of an asthma rehabilitation? Journal of Health Psychology, 1, 1-10. doi: $10.1177 / 1359105316651332$

Ajzen, I., \& Fishbein, M. (1980). Understanding attitudes and predicting social behavior. New York: Prentice-Hall.

Ato, M., López-García, J. J., \& Benavente, A. (2013). Un sistema de 
clasificación de los diseños de investigación en psicología. Anales de Psicología, 29(3), 1038-1059. http://dx.doi. org/10.6018/analesps.29.3.178511

Aujla, N., Walker, M., Sprigg, N., Abrams, K., Massey, A., \& Vedhara, K. (2016). Can illness beliefs, from the commonsense model, prospectively predict adherence to selfmanagement behaviours? A systematic review and meta-analysis. Psychology y Health, 31(8). 931-958. doi: 10.1080/08870446.2016.1153640

Bahçecioğlu, G., \& Çevik-Akyl, R. (2014). Determination of effect on asthma control of illness perception of asthma patients. Acta Medica Mediterránea, 30, 591-600. http:// www.actamedicamediterranea.com/

Beléndez, M., Bermejo, R, M., \& García, M, D. (2005). Estructura factorial de la versión española del Revised Illness Perception Questionnaire en una muestra de hipertensos. Psicothema, 17(2), 318-324. http://www.psicothema.com/ pdf/3106.pdf

Bender, B. G., \& Bender, S. E. (2005). Patient-identified barriers to asthma treatment adherence: Responses to interviews, focus groups, and questionnaires. Immunology and Allergy Clinics of North America, 25(1), 107-130. doi: org 10.1016/j.iac.2004.09.005

Bot, S. D. M., Terwee, C. B., Van der Windt, D. A. W. M., Bouter, L. M., Dekker, J. M., \& De Vet, H. C. W. (2003). Psychometric evaluation of self-report questionnaires: The development of a checklist. In H. J. Adér, y C. Mellenberg (eds.), Proceedings of the second workshop on research methodology (pp. 161-168). Amsterdam: VU University.

Brandes, K., \& Mullan, B. (2014). Can the common-sense model predict adherence in chronically ill patients? A metaanalysis. Health Psychology Review, 8(2), 129-153. doi:10. 1080/17437199.2013.820986

Brink, E. V. A., Alsén, P. I. A., \& Cliffordson, C. (2011). Validation of the Revised IIIness Perception Questionnaire (IPQ-R) in a sample of persons recovering from myocardial infarctionThe Swedish version. Scandinavian Journal of Psychology, 52(6), 573-579. doi: 10.1111/j.1467-9450.2011.00901.x

Broadbent, E., Petrie, K., Main, J., \& Weinman, J. (2006). The brief illness perception questionnaire. Journal of Psychosomatic Research. 60, 631-637. doi:10.1016/j. jpsychores.2005.10.020

Broadbent, E., Wilkes, C., Koschwanez, H., Weinman, J., Norton, S., \& Petrie, K. J. (2015). A systematic review and meta-analysis of the Brief Illness Perception Questionnaire. Psychology y Health, 30(11), 1361-1385. doi: $10.1080 / 08870446.2015 .1070851$

Brzoska, P., Yilmaz, A, Y., Sultanoglu, E., Sultanoglu, B., \& Razum, O. (2012). The factor structure of the Turkish version of the Revised Illness Perception Questionnaire (IPQ-R) in patients with diabetes and cardiovascular disease. BMC Public Health, 12(1), 852. http://www.biomedcentral. com/1471-2458/12/852

Caldera-Alvarado, G., Khan, D. A., Defina, L. F., Pieper, A., \& Brown, E. S. (2013). Relationship between asthma and cognition: The Cooper Center Longitudinal Study. Allergy, 68(4), 545-548. doi:10.1111/all.12125

Cameron, P., \& Moss-Morris, R. (2004). Illness-related cognition and behavior. In: A. A., Kaptein, y J. Weinman. Health
Psychology (pp, 84-110), Oxford: BPS Blackwell.

Chen, S. L., Tsai, J. C., \& Lee, W. L. (2008). Psychometric validation of the Chinese version of the Illness Perception Questionnaire-Revised for patients with hypertension. Journal of Advanced Nursing, 64(5), 524-534. doi: 10.1111/j.1365-2648.2008.04808.x

Cheung, M. M. Y., Saini, B., \& Smith, L. (2017). Drawing asthma: An exploration of patients' perceptions and experiences. Journal of Asthma, 54. 1-10. DOI: 10.1080/02770903.2017.1325492

Chilcot, J., Norton, S., Wellsted, D., \& Farrington, K. (2012). The factor structure of the revised illness perception questionnaire (IPQ-R) in end-stage renal disease patients. Psychology, Health y Medicine, 17(5), 578-588. http:// dx.doi.org/10.1080/13548506.2011.647702

Corral, Y. (2009). Validez y confiabilidad de los instrumentos de investigación para la recolección de datos. Revista Ciencias de la Educación. 19(3), 229-247. Disponible en: http:// servicio.bc.uc.edu.ve/educacion/revista/n33/art12.pdf

Dempster, M., \& McCorry, N. K. (2012). The factor structure of the Revised Illness Perception Questionnaire in a population of oesophageal cancer survivors. Psycho-Oncology, 21(5), 524-530. doi: $10.1002 /$ pon. 1927

DeVellis, R. F. (2012). Scale development: theory and applications. USA: SAGE Publications.

Diefenbach, M. A., \& Leventhal, H. (1996). The common-sense model of illness representation: Theoretical and practical considerations. Journal of Social Distress and the Homeless, 5(1), 11-38. doi: 10.1007/BF02090456

Dirección General de Epidemiología. [DGE] (2015). Anuarios de morbilidad 1984-2015. Recuperado de http://www. epidemiologia.salud.gob.mx/anuario/html/anuarios.html

Ferrando, P. J., \& Anguiano-Carrasco, C. (2010). El análisis factorial como técnica de investigación en psicología. Papeles del Psicólogo, 31(1), 18-33. http://www.redalyc. org/articulo.oa?id $=77812441003$

Foster, J. M., Lavoide, K. L., \& Boulet, L. P. (2011). Treatment adherence and psychosocial factors in severe asthma. En: K. F. Chung, E. H. Bel y S. E. Wenzel (eds) European Respiratory Monograph: Dificult to treat severe asthma, 51 (pp. 28-49). European Respiratory Society. doi: 10.1183/1025448x.erm5110

Giannousi, Z., Manaras, I., Georgoulias, V., \& Samonis, G. (2010). Illness perceptions in Greek patients with cancer: A validation of the Revised-IIIness Perception Questionnaire. Psycho-Oncology, 19(1), 85-92. doi: 10.1002/pon.1538

Global Initiative for Asthma [GINA] (2019). Global strategy for asthma management and prevention. Recuperado de www.ginasthma.org

Guía Mexicana de Asma. [GUIMA] (2017). Guía Mexicana de Asma. Neumología y Cirugía de Tórax, 76, S1, 1-137.

Díaz-Barriga, C. G., \& González-Celis Rangel, A. L. (2019). Propiedades psicométricas del Inventario de Ansiedad de Beck en adultos asmáticos mexicanos. Psicología y Salud, 29(1), 5-16. DOI: https://doi.org/10.25009/pys.v29i1.2563

Halm, E., Mora, P., \& Leventhal, H. (2006). No Symptoms, No Asthma. The acute episodic disease beliefs is associated with poor self-management among inner-city adults with persistent asthma. Chest, 129, 573-580. doi 10.1378/chest.129.3.573 
Hernández-Sampieri, R., Fernández-Collado, C., \& BaptistaLucio, P. (2014). Metodología de la investigación. México: Mc Graw-Hill.

Holmes, E. A., Hughes, D. A., \& Morrison, V. L. (2014). Predicting adherence to medications using health psychology theories: a systematic review of 20 years of empirical research. Value in Health, 17(8), 863-876. doi: .org/10.1016/j.jval.2014.08.2671

Horne, R., \& Hankins, M. (2002). The Medication Adherence Report Scale. Brighton,England: Center for Health Care Research.

Horne, R., \& Weinman, J. (2002). Self-regulation and selfmanagement in asthma: Exploring the role of illness perceptions and treatment beliefs in explaining nonadherence to preventer medication. Psychology y Health, 17(1), 17-32. doi: $10.1080 / 08870440290001502$

Horne, R., Weinman. J., \& Hankins, M. (1999). The Beliefs about Medicines Questionnaire (BMQ): The development and evaluation of a new method for assessing the cognitive representation of medication. Psychology y Health, 14(1), 1-24. doi: 10.1080/08870449908407311

Hurtado-Piña, A. (2017). Validación de un instrumento basado en el Illness Perception Questionnaire-Revised (IPQ-R) para pacientes mexicanos con obesidad (Tesis de maestría inédita), Universidad Iberoamericana, México. http://ri.ibero.mx:8080/viewer/?code $=016446 \#$

Instituto Nacional de Enfermedades Respiratorias. [INER] (2018). Informe anual 2017. Recuperado de www.iner. salud.gob.mx

Kucukarslan, S. N. (2012). A review of published studies of patients' illness perceptions and medication adherence: Lessons learned and future directions. Research in Social and Administrative Pharmacy, 8(5), 371-382. doi:10.1016/j. sapharm.2011.09.002

Kucukarslan, S. N., Lee, K. S., Patel, T. D., \& Ruparelia, B. (2013). An experiment using hypothetical patient scenarios in healthy subjects to evaluate the treatment satisfaction and medication adherence intention relationship. Health Expectations, 18(5), 1291-1298. doi: 10.1111/hex.12103

Kucukarslan, S. N., Plumley, D., Chang, A., \& Ueda, A. (2014). Intending to adhere or to not adhere: Results from an experiment in healthy subjects testing illness perceptions and behavioral intentions in asthma. Research in Social and Administrative Pharmacy, 10(1), 239-245. DOI: http:// dx.doi.org/10.1016/j.sapharm.2013.05.001

Leventhal, H., Breland, J. Y., Mora, H., \& Leventhal, E. (2010). Lay representations of Illness and treatment: A Framework for Action. In A. Steptoe (ed.), Handbook of Behavioral Medicine (pp, 137-154). USA: Springer Science-Business Media. Doi 10.1007/978-0-387-09488-5_11

Leventhal, H., Weinman, J., Leventhal, E., \& Phillips, A. (2008). Health Psychology: The search for pathways between behavior and health. Annual Review of Psychology. 59, 477-505. doi: 10.1146/annurev.psych.59.103006.093643

Lloret-Segura, S., Ferreres-Traver, A., Hernández-Baeza, A., \& Tomás-Marco, I. (2014). El análisis factorial exploratorio de los ítems: Una guía práctica, revisada y actualizada. Anales de Psicología, 30(3), 1151-1169. http://dx.doi. org/10.6018/analesps.30.3.199361.
Lozano, L. M., García-Cueto, E., \& Muñiz, J. (2008). Effect of the number of response categories on the reliability and validity of rating scales. Methodology, 4(2), 73-79. doi 10.1027/1614-2241.4.2.73

Lugo-González, I, V., Reynoso-Erazo, L., \& Fernández-Vega, M. (2014). Percepción de enfermedad, depresión, ansiedad y control del asma: Una primera aproximación. Neumología y Cirugía de Tórax. 73(2), 114-121. http:// new.medigraphic.com/

Lugo-González, I, V., \& Vega-Valero, C, Z. (2020). Propiedades psicométricas de la Medication Adherence Report ScaleAsthma (MARS-A) en adultos mexicanos con asma. Psicología y Salud. 30(2).

McAndrew, L. M., Musumeci-Szabó, T. J., Mora, P. A., Vileikyte, L., Burns, E., Halm, E. A., Leventhal, E., \& Leventhal, $H$. (2008). Using the common-sense model to design interventions for the prevention and management of chronic illness threats: From description to process. British Journal of Health Psychology, 13(2), 195-204. doi:10.1348/135910708X295604

Mora, P., \& McAndrew. (2013). Common-sense model of self-regulation. En: M.D. Gellman y J.R. Turner (eds.) Encyclopedia of Behavioral Medicine (pp. 460-467). New York: Springer. doi 10.1007/978-1-4419-1005-9

Moon, Z., Moss-Morris, R., Hunter, M. S., \& Hughes, L. D. (2017). Measuring illness representations in breast cancer survivors (BCS) prescribed tamoxifen: Modification and validation of the Revised IIIness Perceptions Questionnaire (IPQ-BCS). Psychology y Health, 32(4), 439-458. http:// dx.doi.org/10.1080/08870446.2016.1275629

Moss-Morris, R., Weinman, J., Petrie, K., Horne, R., Cameron, L., \& Buick, D. (2002). The Revised Illness Perception Questionnaire (IPQ-R). Psychology y Health. 17(1), 1-16. doi: 10.1080/08870440290001494

Nathan, R. A., Sorkness, C. A., Kosinski, M., Schatz, M., Li, J. T., Marcus, P., Murray, J., \& Pendergraft, T. B. (2004). Development of the asthma control test: a survey for assessing asthma control. Journal of Allergy and Clinical Immunology, 113(1), 59-65. DOI: https://doi.org/10.1016/j. jaci.2003.09.008

Osborne,J.W.(2014). BestPracticesinExploratory FactorAnalysis. Scotts Valley, CA: CreateSpace Independent Publishing. ISBN-13:978-1500594343, ISBN-10:1500594342.

Østrem, A., \& Horne, R. (2015). Reducing asthma attacks: consider patients' beliefs. Primary Care Respiratory Medicine, 25, 15021. doi:10.1038/npjpcrm.2015.21

Pacheco, V., Viladrich, C., Pujol, E., Cabezas, C., Núñez, M., Roura, P., Núñez, E., \& Del Val, J. L. (2012). Percepción en enfermedades crónicas: Validación lingüística del Illness Perception Questionnaire Revised y del Brief Illness Perception Questionnaire para la población española. Atención Primaria. 44(5), 280-287. doi:10.1016/j. aprim.2010.11.022

Pedrosa, I., Juarros, J., Robles, A., Basteiro, J., \& García, E. (2015). Pruebas de bondad de ajuste en distribuciones simétricas ¿Qué estadístico utilizar? Universitas Psychologica, 14(1), 245. doi:10.11144/Javeriana.upsy14-1.pbad

Petrie, K., \&Weinman,J. (2006). Why illness perceptions matter. Clinical Medicine, 6(6), 536-539. doi: 10.7861/clinmedicine.6-6-536 
Petrie, K., \& Weinman, J. (2012). Patients' perceptions of their illness: The dynamo of volition in health care. Current Directions in Psychological Science, 21(1), 60-65. doi: $10.1177 / 0963721411429456$

Ponieman, D., Wisnivesky, J. P., Leventhal, H., Musumeci-Szabó, T. J., \& Halm, E. A. (2009). Impact of positive and negative beliefs about inhaled corticosteroids on adherence in inner-city asthmatic patients. Annals of Allergy, Asthma y Immunology, 103(1), 38-42. doi: 10.1016/S1081$1206(10) 60141-X$.

Reyes-Lagunes., I. (2011). Conceptualización y desarrollo de la etnopsicometría en México. En A. Domínguez (comp.), Letras introductorias a la psicología cultural, transcultural y etnopsicología (pp. 279-302). México: Universidad Iberoamericana.

Reyes-Lagunes, I., y García \& Barragán, L. (2008). Procedimiento de validación psicométrica culturalmente relevante: Un ejemplo. La Psicología Social en México, XII, 625-630.

Secretaría de Salud. [SSA] (2016). Siete por ciento de la población en México padece asma. Recuperado de: https://www. gob.mx/salud/prensa/siete-por-ciento-de-la-poblacionen-mexico-padece-asma

Sofianou, A., Martynenko, M., Wolf, M., Wisnivesky, J., Krauskopf, K., Wilson, E., Sanghavi, M., Leventhal, H., Halm, M., \& Federman, A. (2013). Asthma beliefs are associated with medication adherence in older asthmatics. Journal of General Internal Medicine, 28(1), 67-73. doi: 10.1007/ $\underline{\text { s11606-012-2160-z }}$
Unni, E., \& Shiyanbola, O. O. (2016). Clustering medication adherence behavior based on beliefs in medicines and illness perceptions in patients taking asthma maintenance medications. Current Medical Research and Opinion, 32(1), 113-121. doi: 10.1185/03007995.2015.1105204

Van Ittersum, M. W., Van Wilgen, C. P., Hilberdink, W. K. H. A., Groothoff, J. W., \& Van Der Schans, C. P. (2009). Illness perceptions in patients with fibromyalgia. Patient Education and Counseling, 74(1), 53-60. dpi:10.1016/j. pec.2008.07.041

Weinman, J., Petrie, K., Moss-Morris, R., \& Horne, R. (1996). The Illness Perception Questionnaire: A new method for assessing the cognitive representation of illness. Psychology y Health, 11(1), 431-45. doi: 10.1080/08870449608400270

Wittkowski, A., Richards, H. L., Williams, J., \& Main, C. J. (2008). Factor analysis of the Revised Illness Perception Questionnaire in adults with atopic dermatitis. Psychology, Health and Medicine, 13(3), 346-359. doi: 10.1080/13548500701487697

Wu, X., Lau, J. T., Mak, W. W., Gu, J., Mo, P. K., \&Wang, X. (2018). How newly diagnosed HIV-positive men who have sex with men look at HIVIAIDS-Validation of the Chinese version of the revised illness perception questionnaire. BMC Infectious Diseases, 18(1), 1-10. doi:10.1186/s12879-017-2902-y 


\section{Meta-Análisis del Artículo}

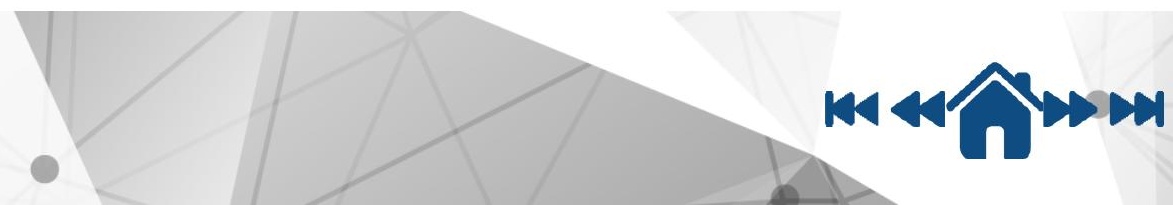




\section{Dimensión Cuantitativa}

\section{Perfil de Evaluación entre pares}
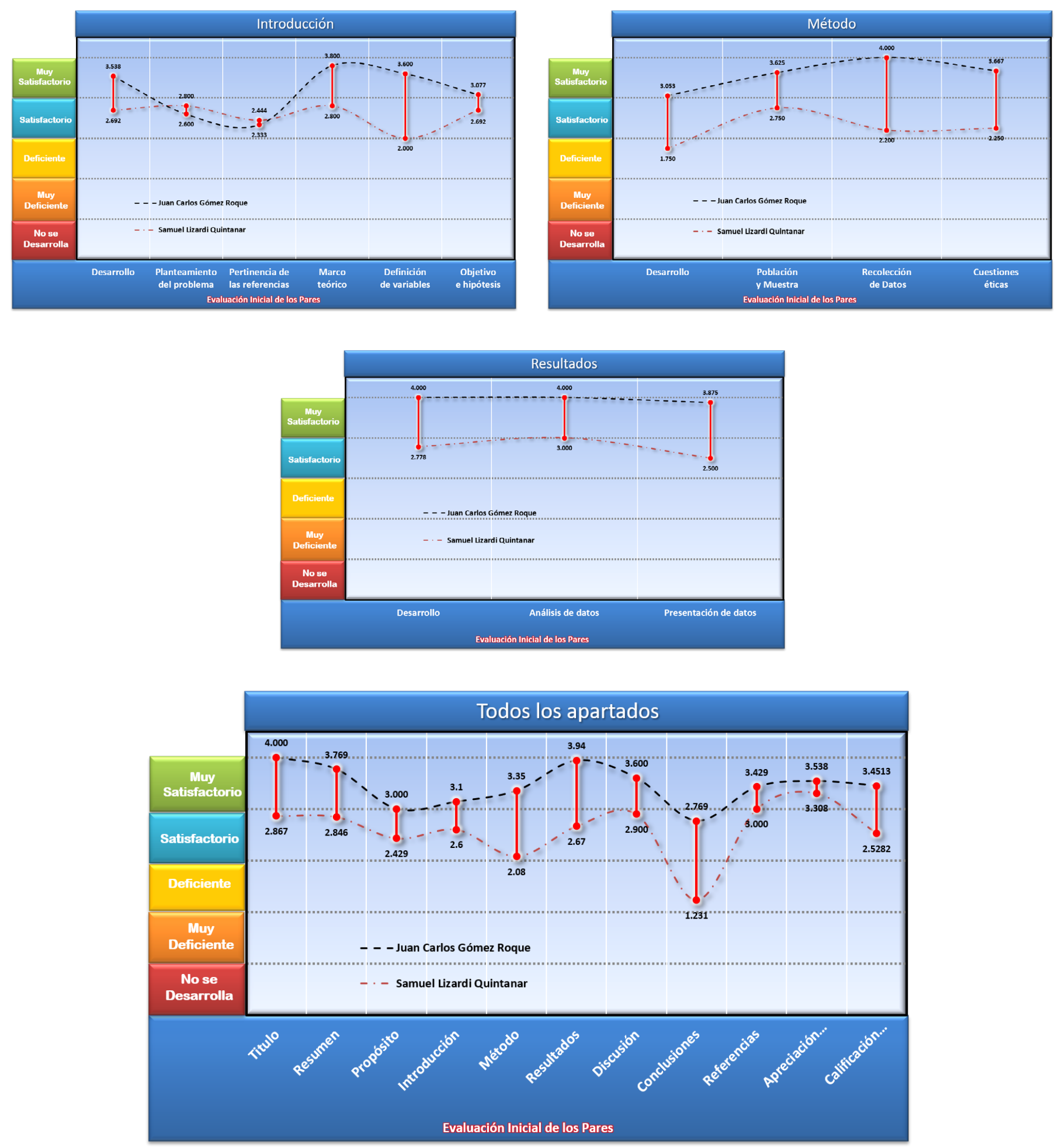


\section{Índice de Concordancia}

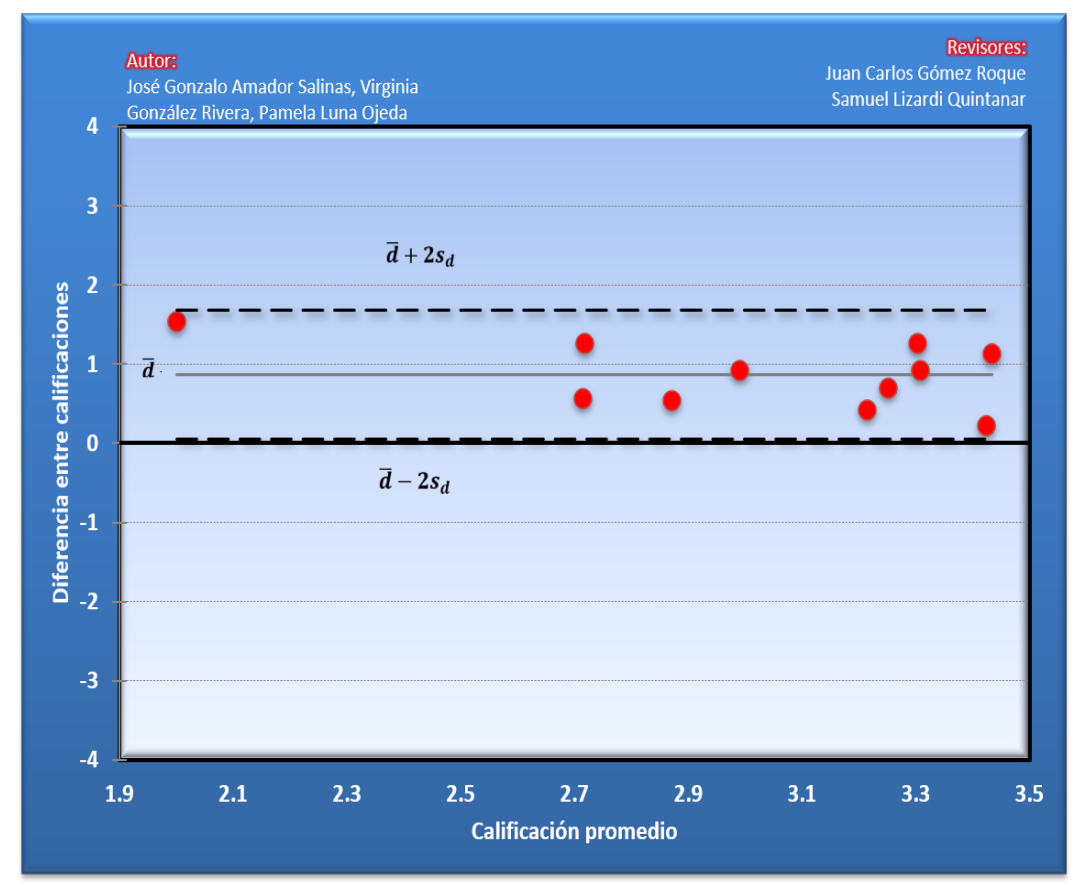

Índice de Acuerdo

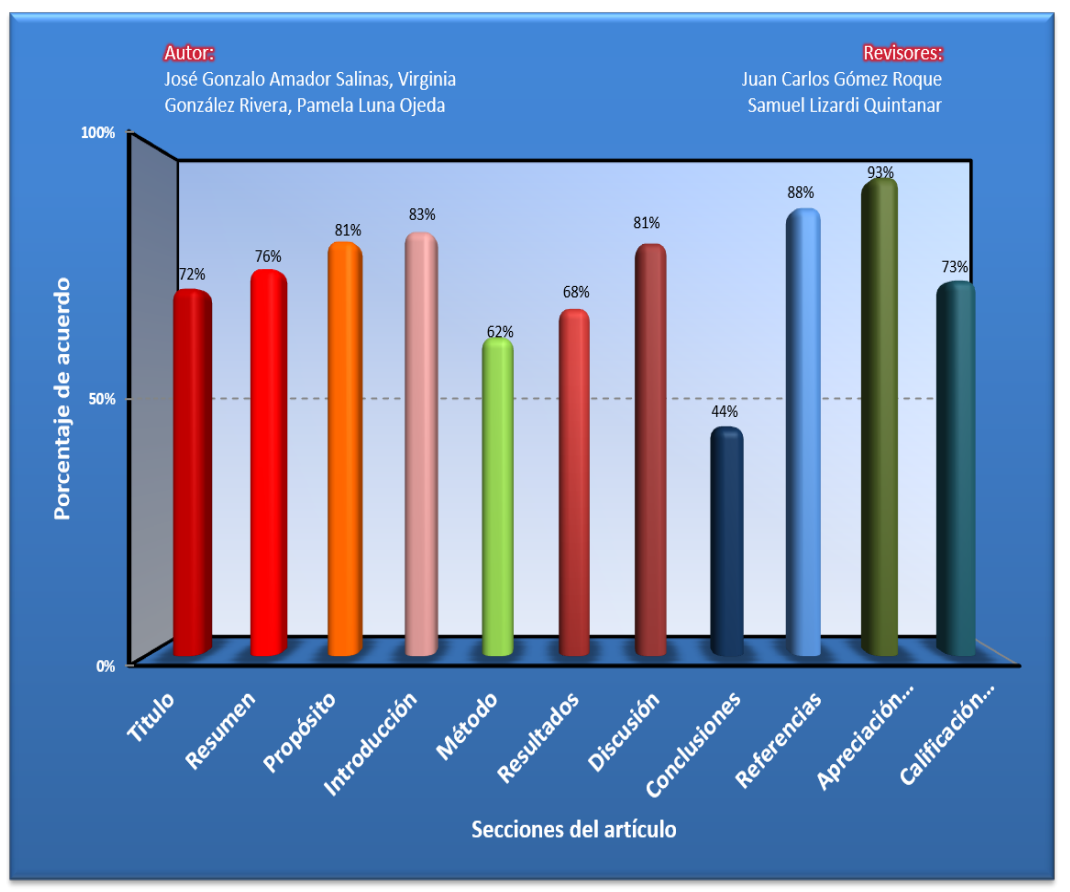




\begin{tabular}{|c|c|}
\hline Revisor 1 & Revisor 2 \\
\hline Nazira Calleja Bello & Carlos Prado Romero \\
\hline \multicolumn{2}{|l|}{ Título/Autoría } \\
\hline $\begin{array}{l}\text { El título es adecuado. Sin embargo, me parece que sería muy conve- } \\
\text { niente incluir el título de la escala en español. }\end{array}$ & Ninguna \\
\hline \multicolumn{2}{|l|}{ Resumen } \\
\hline 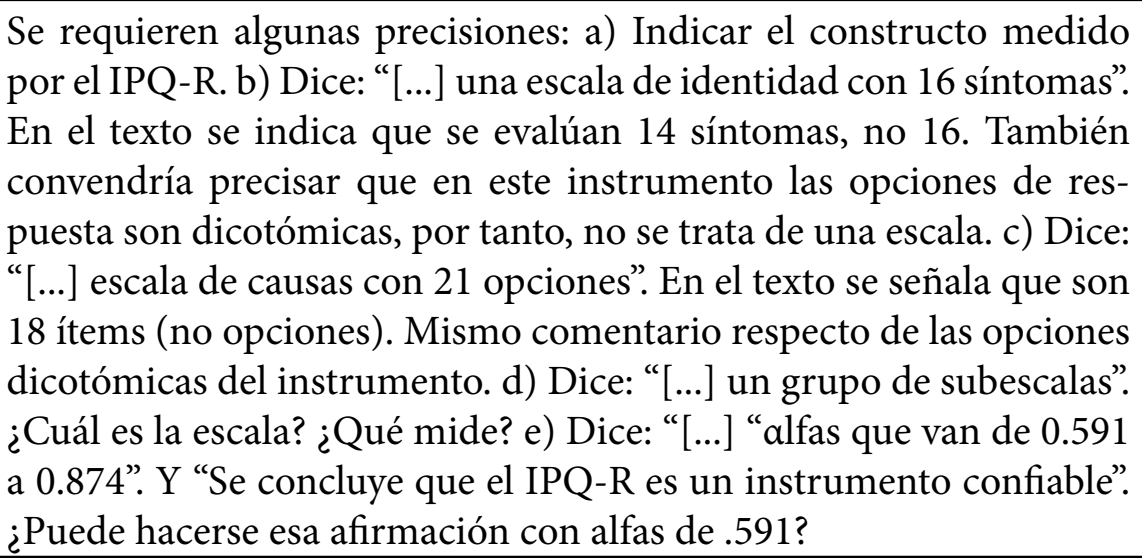 & Ninguno \\
\hline \multicolumn{2}{|l|}{ Próposito del Estudio } \\
\hline $\begin{array}{l}\text { Se sugiere ser más específicos en la redacción del propósito del estudio. } \\
\text { Dice: “[...] evaluar las propiedades psicométricas de la versión en espa- } \\
\text { ñol”. ¿Cuáles propiedades psicométricas? ¿De qué versión en español? }\end{array}$ & Ninguno \\
\hline \multicolumn{2}{|l|}{ Introducción } \\
\hline $\begin{array}{l}\text { a) Puesto que las creencias sobre la enfermedad constituyen la variable } \\
\text { básica del estudio, se sugiere ampliar la información respecto de éstas y } \\
\text { de su efecto sobre otras variables, así como presentar a los autores co- } \\
\text { rrespondientes de cada hallazgo, evitando la larga lista de citas. b) Sería } \\
\text { necesario ampliar la información psicométrica del IPQ-R, objeto del } \\
\text { trabajo: la razón de esta versión revisada y sus ventajas respecto de la } \\
\text { versión original, la definición de los constructos que mide, la población } \\
\text { con la que validó, su validación de contenido y de criterio y predictiva, } \\
\text { sus índices de estabilidad temporal, etc. c) En la Introducción dice: “[...] } \\
\text { conformado por } 70 \text { ítems divididos en nueve subdimensiones", en tanto } \\
\text { que en la sección de Método se señala: “[...] conformado por } 70 \text { ítems } \\
\text { divididos en tres escalas". d) Justificar por qué se eligió el IPQ-R sobre el } \\
\text { BIPQ. e) Describir las versiones del instrumento que se han validado en } \\
\text { español y justificar la elección de la versión de Beléndez et al. (2005). f) } \\
\text { Ampliar la investigación respecto de la asociación de las creencias sobre } \\
\text { la enfermedad con las variables utilizadas para la validez de criterio, en } \\
\text { términos de la red nomológica del constructo. }\end{array}$ & Ninguno \\
\hline
\end{tabular}




\section{Revisor 1}

Revisor 2

\section{Método}

a) Para facilitar la compresión de las características de los participantes (no sujetos), se sugiere utilizar una tabla para los datos sociodemográficos y de variables relativas a la enfermedad, sólo con valores relativos (no absolutos). Incluir rango de edad y de tiempo de evolución de la enfermedad. b) Señalar que se trata de pacientes atendidos y abordados en una clínica pública (sin mencionar el nombre de ésta).. c) ¿Los criterios de exclusión son sólo la negación de los de inclusión? Los criterios de eliminación parecerían obvios. d) Más específicamente, se trata de un diseño de tipo instrumental. Consultar a Ato, López y Benavente, 2013. e) ¿Todos los datos contenidos en la Cédula de datos sociodemográficos y clínicos se encuentran incluidos en la descripción de los participantes? f) Es necesario definir cada una de las variables medidas por el IPQ-R. g) Valdría la pena revisar la denominación de la primera escala, ya que "Identidad" no señala claramente el contenido de la escala, que es "Síntomas". h) Dice: “[...] y para la tercera escala de 0.23 a 0.86 ". Revisar el índice ".23"; seguramente hay un error, y sí no lo es, incluir las explicaciones del autor. i) De acuerdo con las normas APA, no incluir el 0 en una cifra cuyo valor máximo es 1 , como es el caso de los índices alfa. j) Puesto que se usó una versión del IPQ-R validad en España, indicar los procedimientos de adaptación al español hablado en México. k) Dice: “[...] se redujeron a cuatro las opciones de respuesta para evitar respuestas centrales". Esta afirmación no es correcta. La reducción en el número de opciones de respuesta reduce la variabilidad y afecta sensiblemente la calidad de la medición. Consultar: Simms, L. J., Zelazny, K., Williams, T. F., \& Bernstein, L. (2019). Does the number of response options matter? Psychometric perspectives using personality questionnaire data. Psychological Assessment, 31(4), 557-566. doi: $10.1037 /$ pas0000648 l) $\dot{i}$ Los índices de confiabilidad que se indican del BMQ son los reportados por el autor original o corresponden a la validación en México? ¿Cuál es el índice de la escala de daño? m) Respecto del MARS-A dice: "[...] cuenta con datos adecuados de validez". Especificarlos. n) Indicar la versión que se aplicó del ACT. y ampliar la información sobre sus características psicométricas. o) Dice: "Dado que la escala de identidad y la escala de causas consideran identificar los síntomas atribuibles a la enfermedad y las posibles causas de esta, los análisis necesarios únicamente son de tipo descriptivo y resulta no necesario obtener alguna propiedad psicométrica de estas escalas." Fundamentar con argumentos de autores reconocidos esta aseveración. p) Fundamentar la utilización del método de Ejes Principales y la rotación Promax en el análisis factorial exploratorio. ¿Por qué no usar correlaciones policóricas, dada la no normalidad de las variables? p) Dice: "[...] y para la agrupación de los ítems a los factores se tomó el criterio de $\geq .4$ ". Explicar y sustentar esta frase. q) Es fundamental efectuar el análisis factorial confirmatorio del instrumento. r) Indicar la razón de la utilización de índice Rho. s) Señalar la dirección y magnitud aproximada esperadas de los índices de correlación de las subescalas del IPQ-R con las escalas y subescalas planteadas como evidencia de validez de criterio. t) ampliar la descripción del procedimiento de aplicación de los instrumentos. Eliminar el nombre del instituto en el que se efectuó el estudio. ¿El consentimiento informado se entregó al final no al inicio de la aplicación? 


\section{Revisor 1}

Revisor 2

\section{Resultados}

Se sugiere presentar las escalas y subescalas siempre en el mismo orden y con el mismo nombre. Escala de identidad: a) Se sugiere que los datos de la tabla 1 se presenten gráficamente y en orden decreciente, representando sólo los porcentajes de "si" de los síntomas experimentados y de los identificados, ya que el resto de los datos es redundante. b) Sería muy esclarecedor indicar cuáles de los síntomas corresponden efectivamente a la enfermedad, de acuerdo con los reportes clínicos. c) Se reporta la media, la desviación estándar y el rango para "identidad de síntomas". ¿Corresponde a síntomas experimentados o identificados, o a una combinación de ambos? d) Se señala que "[...] en esta escala se les pide a los pacientes que mencionen aquellos síntomas que identifiquen con su enfermedad y que no estén mencionados". ¿Esta indicación se encuentra también en la escala original? Incluir los datos correspondientes. e) Obtener la correlación entre los síntomas experimentados y los identificados. Con base en este resultado, analizar en la discusión, la pertinencia de evaluar ambas. g) Puede obtenerse la confiabilidad para datos dicotómicos. Escala de causas a) Los autores de esta escala la construyeron y validaron con opciones de respuesta escaladas y con cuatro dimensiones o factores. Se sugiere que se efectúen los análisis psicométricos correspondientes para validar esta estructura factorial, lo cual aportarían valiosa información sobre las variables latentes que subyacen a las causas que los pacientes atribuyen a su enfermedad. Ello no implicaría que, pudieran detectarse las causas "puntuales" utilizando, no sólo porcentajes, sino medias de los reactivos. b) Dice: "[...] se les pidió que ordenaran en importancia las tres primeras causas de su enfermedad". ¿Esta instrucción se encontraba también en el original? ¿Cuáles fueron los datos obtenidos? ¿Arrojó información adicional a la que se puede derivar de los reactivos originales? Escala de ¿Creencias sobre la enfermedad? (Se entiende que el IPQ-R es un inventario que incluye no sólo esta escala, sino también las dos anteriores, por lo que no puede usarse esta denominación sólo para ésta). a) ¿Que significa la frase: "[...] el puntaje promedio para cada ítem fue diverso debido a que cada una de las subescalas orienta en dar respuestas diferentes"? b) Describir cómo se formaron los grupos alto y bajo para la discriminación de reactivos. Dice: "[...] se contrastaron los percentiles uno y cuatro". ¿Será más bien que con base en los cuartiles 1 y 3 se formaron los grupos alto y bajo? c) En la tabla 3, en lugar de las frecuencias de respuesta para cada opción, proporcionar los porcentajes correspondientes. d) Valdría la pena señalar que en las dimensiones: Control personal, Control del tratamiento y Coherencia (entendimiento de la enfermedad), los puntajes altos corresponden a creencias favorables sobre el control de la enfermedad, en tanto que en las dimensiones: Temporalidad (cronicidad de la enfermedad), Ciclicidad, Consecuencias (negativas) y Representación emocional (impacto negativo), los puntajes altos indican creencias desfavorables sobre la enfermedad. e) No es necesario incluir el primer párrafo del "Análisis de confiabilidad y validez del constructo del IPQ". f) Dice: “[...] el IPQ-R en población mexicana con asma [...] está conformado por cuatro factores [...] Además, cuenta con tres indicadores: 1) coherencia con tres ítems; 2) curso cíclico con dos ítems; y 3) control del tratamiento con tres ítems." Fundamentar por qué se toma una dimensión con tres reactivos como indicador. g) Si se partió de la no normalidad de los datos, habría que calcular no el alfa de Cronbach, sino el omega de McDonald. h) En la tabla 4 incluir la numeración de los reactivos. i) Efectuar y reportar el análisis factorial confirmatorio. j) Reportar las correlaciones inter-factor. Evidencia de validez convergente y divergente: a) Presentar las correlaciones en una tabla. Incluir todas las correlaciones de los puntajes de los siete factores de la Escala de Creencias, de las dos de Identidad (Síntomas experimentados y Síntomas identificados) y de las de Causas contra los de los tres del BMQ (Necesidad, Preocupación y Daño), del MARS-A y del ACT. b) Incluir las medidas descriptivas de todos los factores y escalas.

Una vez realizado el AFE con resultados favorables, enriquecería el trabajo presentar un AFC para aportar mayores evidencias sobre la validez de la escala. 
Revisor 1
Revisor 2

\section{Discusión}

a) Cuando se afirma que se eliminaron o incluyeron síntomas o causas, ¿se quiere decir que se propone que en futuras aplicaciones de la escala se efectúen tales cambios? b) ¿Por qué se consideró pertinente agrupar en un solo síntoma "cansancio" y "debilidad", siendo que los porcentajes para cada uno difieren en cerca de 20 puntos? c) Indicar la razón por la que se propone incluir tres síntomas más a la escala: dolor de cabeza, ansiedad y tristeza. d) Dice: "[...] sabiendo que la segunda respuesta es la que se contabiliza en la escala". Convendría hacer este señalamiento desde la descripción del instrumento. e) Dice: "[...] y ambas opciones complejiza la forma de respuesta". Aclarar esta afirmación. f) Dice: "[...] la pregunta es si se desea saber qué es lo que creen que causó su enfermedad o con qué causas están más de acuerdo". Probablemente la percepción de las causas de la enfermedad no se dé en términos absolutos, sino de grado: algunos elementos podrían considerarse más relevantes como causas, en comparación con otros que también se creen que intervienen, pero en menor grado. Quizá los autores de la escala consideraron que la forma de medición escalada ofrecería más precisión en la evaluación de esta variable. Habría que incluir este punto en la discusión. g) Dice: "[...] fumar estar expuesto al humo de tabaco y fumar se incluyó en una misma causa". Esta frase resulta confusa. Señalar la razón por la que se fusionaron. h) En el párrafo en el que se discuten los índices de confiabilidad, eliminar las cifras y señalar específicamente cuáles son las discrepancias con la versión original. i) Dice: "[...] emplearon técnicas de factorización basados en el análisis de componentes principales con rotación varimax, lo cual es considerado inadecuado ya que este tipo de factorizaciones no se dirigen a identificar una estructura factorial, sino por el contrario, a reducir el número de variables." En ambos casos el propósito es reducir el número de variables; la diferencia entre ambos tiene que ver con los términos de error. j) Dice "En este sentido, dado que en el presente estudio no se identificó normalidad en los datos del IPQ-R, se procedió a realizar el análisis basado en ejes principales, el cual se recomiendo cuando los datos no son normales". Cuando los datos de asimetría y curtosis indican anormalidad extrema, se recomienda el uso de correlaciones policóricas. k) Dice: "[...] seis de ellos, fueron ítems negativos". Cinco de estos reactivos incluyen la palabra "no", cuyo uso es altamente desalentado en la construcción de escalas. Quizá podría sugerirse que en una futura aplicación se re-frasearan estos reactivos y probarlos, antes de decidir definitivamente su eliminación. 1) En el párrafo de la validez convergente eliminar las cifras. Incluir el análisis de todas las correlaciones pertinentes. m) Dice: "[...] de hecho, se ha cuestionado no sólo el papel de las creencias sobre la enfermedad como variables asociadas a la adherencia”. Llama la atención que se haya elegido la adherencia como variable para obtener evidencia de validez de criterio, siendo que no se ha establecido claramente su relación con las creencias. n) Es más sorprendente aún que se haya trabajado tan arduamente en la validación de un instrumento que ha sido derivado de un modelo (MSCRE), el cual ha recibido críticas sustentadas respecto de la forma en que han sido elaborados los reactivos. o) Dice: “[...] consideran necesario incluir las creencias sobre el tratamiento [...] al MSCRE". ¿No están incluidas en el modelo, pero sí en el instrumento? p) Eliminar del último párrafo las cifras. q) Dice "[...] se observa claramente que las correlaciones entre creencias sobre el asma y sobre el tratamiento de control son mejores" ¿Tratamiento de control o Control del tratamiento? ¿Mejores o más altas? r) Incluir la discusión sobre las características de la muestra.
Las limitaciones y áreas de oportunidad del presente estudio son evidenciadas en el apartado de las conclusiones, no en la discusión 


\begin{tabular}{|l|l|}
\hline \multicolumn{1}{|c|}{ Revisor 1 Conclusiones } & \multicolumn{2}{|c|}{ Revisor 2 } \\
\hline $\begin{array}{l}\text { a) El análisis de las diferencias de género en la prevalencia de la en- } \\
\text { fermedad correspondería más apropiadamente a la sección de Dis- } \\
\text { cusión. b) Si hay mayor prevalencia de la enfermedad entre hombres } \\
\text { y mujeres, ¿por qué “puede representar un problema” para la vali- } \\
\text { dación del instrumento? c) Las conclusiones deberían enriquecerse } \\
\text { sustancialmente. }\end{array}$ & Ninguno \\
\hline \multicolumn{1}{|c|}{ Referencias } \\
\hline $\begin{array}{l}\text { Revisar la inclusión de todos los autores citados en el texto. Por } \\
\text { ejemplo, Levesque, Li \& Pahal (2012) no se encuentra en el listado } \\
\text { de Referencias. }\end{array}$ & Ninguno \\
\hline
\end{tabular}


